\title{
RAMIFICATION THEORY AND FORMAL ORBIFOLDS IN ARBITRARY DIMENSION
}

\author{
MANISH KUMAR
}

\begin{abstract}
Formal orbifolds are defined in higher dimension. Their étale fundamental groups are also defined. It is shown that the fundamental groups of formal orbifolds have certain finiteness property and it is also shown that they can be used to approximate the étale fundamental groups of normal varieties. Etale site on formal orbifolds are also defined. This framework allows one to study wild ramification in an organised way. Brylinski-Kato filtration and $l$-adic sheaves in these contexts are also studied.
\end{abstract}

\section{INTRODUCTION}

Let $X$ be a normal variety. Tamely ramified covers of $X$ are easy to understand. This is because locally these covers are determined by the ramification index. But this is not at all true for wildly ramified covers of $X$. This makes the study of wild ramification very tedious. In this paper we define a structure $P$ on $X$ called the branch data (see Section 3). When $X$ is a curve over an algebraically closed field then these objects were defined and studied in $\mathrm{KP}$. For a closed point $x \in X$, $P(x)$ is just a finite Galois extension of the fraction field of $\widehat{\mathcal{O}}_{X, x}$ like in $\mathrm{KP}$ but for higher dimensional $X$ the definition of branch data is slightly more intricate (see Definition 3.3). The pair $(X, P)$ is called a formal orbifold. These objects provide a framework to study wild ramification in a relatively organised way.

Section 2 develops ramification theory for excellent normal rings $R$ of arbitrary dimension. Some variants of which can be found in $\mathrm{Abh}$. Lemma 2.9 is the main result of this section which generalizes [Kum, Lemma 3.1] to higher dimension. In section 3 branch data on $X$ is defined and some of its basic properties are studied. Every finite dominant separable morphism from $Y \rightarrow X$ give rise to a Branch data on $X$ (Proposition 3.5). Formal orbifolds are defined in section 4 to be normal varieties $X$ together with a branch data $P$. Coverings, étale and flat covers of formal orbifolds are also studied in this section. Fiber products of coverings and their properties are also discussed. It is shown that fiber product of étale coverings of formal orbifolds is an étale covering (Corollary 4.18).

The étale fundamental group of formal orbifolds are defined in section 5 . Their basic properties which are analogous to fundamental groups of schemes are also proved. The following nice property about them are proved.

Theorem 1.1. (see Corollary 5.8) Let $(X, P)$ be a connected formal orbifold with $X$ projective over $\operatorname{Spec}(k)$ where the field $k$ is such that the absolute Galois group $G_{k}$ has finite rank then $\pi_{1}(X, P)$ has finite rank.

It is also shown that they approximate the fundamental group of a normal variety. 
Theorem 1.2. (see Theorem 5.4) Let $X$ be a normal connected variety over a perfect field or $\operatorname{Spec}(\mathbb{Z})$ and $U$ an open subset of $X$. Then $\pi_{1}(U) \cong \lim \pi_{1}(X, P)$ where $P$ runs over all the branch data on $X$ such that $\mathrm{BL}(P) \subset X \backslash U$.

The above two results applied together implies that $\pi_{1}(U)$ for $U$ quasiprojective variety over $k$ is the inverse limit of finite rank profinite groups which have a geometric interpretation.

In section [6 étale site, presheaves and sheaves on formal orbifolds are defined. Structure sheaf of formal orbifolds are defined and their stalks have been computed. Note that a formal orbifold $(X, O)$ where $O$ is the trivial branch data is essentially the same as the normal variety $X$. In particular the étale site on $(X, O)$ is the same as the small étale site on $X$ (and hence $\pi_{1}(X, O)=\pi_{1}(X)$ ). A geometric formal orbifold is a formal orbifold $\mathcal{X}=(X, P)$ which admits a finite Galois étale morphism $f:(Y, O) \rightarrow(X, P)$ (in the sense of formal orbifold) for some $Y$ with trivial branch data $O$. In such a situation cohomology groups of $\mathcal{X}$ can be computed using the cohomology groups of $Y$ by a spectral sequence (Proposition 6.6).

In section 7 using swan conductors of local fields defined by Kato and Matsuda a divisor $S w(X, P)$ is associated to branch data $P$ on $X$ whose branch locus has irreducible components of pure codimension one in $X$. The abelianized fundamental group parameterizing covers with certain bounded ramification $\pi_{1}^{a b}(X, D)$ defined by Kerz and Saito in [KS2] and [KS1] for an effective Cartier divisor $D$ on $X$ is also shown to be the inverse limit of $\pi_{1}^{a b}(X, P)$ where $P$ varies over branch data with $S w(X, P) \leq D$. This leads to questions such as whether some version of class field theory holds for formal orbifolds (7.5) and whether a version of Lefschetz's theorem for fundamental groups is true for formal orbifolds (8.1).

In section 8 we make some progress towards the later. Note that Lefschetz theorem for fundamental group is false for affine varieties in positive characteristic. Though Lefschetz theorem holds if one restricts to prime-to-p étale fundamental group or more generally tame fundamental group (EKi]). To allow wild ramification as well, one has to bound the ramification in some sense. In KS1 Kerz and Saito prove Lefschetz theorem for $\pi_{1}^{a b}(X, D)$. The definition of $\pi_{1}^{a b}(X, D)$ is the dual of the first cohomology group and their proof is cohomological in nature. Here we follow the method adopted in [SGA2, Chapter XII], GM, 4.3] and [EKi]. More precisely, we show the following results.

Proposition 1.3. (Proposition 8.13) Let $(X, P)$ be a projective formal orbifold over a perfect field $k$ of dimension at least two, $Y$ be a normal hypersurface of $X$ which intersects $\mathrm{BL}(P)$ transversally and the divisor $[Y]$ is a very ample divisor on $X$. Then the functor $\operatorname{Cov}(X, P) \rightarrow \operatorname{Cov}\left(\widehat{X}_{Y}, \hat{P}\right)$ is fully faithful. Here $\widehat{X}_{Y}$ is the completion $X$ along $Y$ and $\hat{P}$ is the branch data on $\widehat{X}_{Y}$ induced from $P$.

Theorem 1.4. (Theorem8.11) Under the Hypothesis 8.5, the functor $\operatorname{Cov}\left(\widehat{X}_{Y}, \hat{P}\right) \rightarrow$ $\operatorname{Cov}\left(Y, P_{\mid Y}\right)$ is an equivalence.

The Hypothesis 8.5 essentially says that the formal orbifold $(X, P)$ is geometric, irreducible, smooth and projective over $k ; Y$ is a smooth irreducible hypersurface of $X$ not contained in $\mathrm{BL}(P)$ such that for any point $y \in \mathrm{BL}(P) \cap Y$ there is only one point lying above it in the cover obtained from taking the normalization of a formal local neighbourhood $\widehat{U}_{y}$ of $y$ in $P(y, U)$. As a consequence of the above two results we get the following: 
Corollary 1.5. (Corollary 8.15) Let $(X, P)$ be a smooth irreducible projective formal orbifold over a perfect field $k$ of dimension at least two. The functor from $\operatorname{Cov}(X, P) \rightarrow \operatorname{Cov}\left(Y, P_{\mid Y}\right)$ is fully faithful if $Y$ satisfies Hypothesis 8.5. In particular the map natural map $\pi_{1}\left(Y, P_{\mid Y}\right) \rightarrow \pi_{1}(X, P)$ is an epimorphism.

The above is a wild analogue of [GM, 4.3.7].

In section $9 l$-adic sheaves on $X^{o}$ with normal compactification $X$ is analyzed in the language of formal orbifolds. It is shown that a locally constant sheaf $\mathcal{F}^{o}$ on $X^{o}$ extends to a locally constant sheaf $\mathcal{F}$ on a geometric formal orbifold $(X, P)$ for some branch data $P$ with branch locus contained in the boundary. It is also shown that if the ramification of $\mathcal{F}^{o}$ is bounded by a Galois étale cover $f: Y^{o} \rightarrow X^{o}$ (in the sense of [Dri], EKe, Esn, etc.) then one could choose $P$ such that the branch data $f^{*} P$ on the normalization $Y$ of $X$ in $k\left(Y^{o}\right)$ is curve-tame. This allows us to observe that a lisse $l$-adic sheaf on $X^{o}$ has ramification bounded by a morphism $f$ then it has ramification bounded by any other morphism whose branch data is same as the branch data associated to $f$ up to tame part (Corollary 9.10).

\section{ACKNOWLEDGEMENTS}

The author thanks Suresh Nayak for some useful discussions and an anonymous referee for useful suggestions.

\section{Local Theory}

We begin with some results on ramification theory. Proposition 2.2 and 2.6 below are variants of results in Section 7 of [Abh] where the treatment is for arbitrary normal local domains and hence the comparison with completion of the rings are missing.

For a ring $R$ and a prime ideal $P$, let $\widehat{R}^{P}$ denote the $P$-adic completion of $R$. For a local ring $R, \widehat{R}$ denotes the completion of $R$ at the maximal ideal. For an integral domain $R, \mathrm{QF}(R)$ denotes the field of fractions (=quotient field) of $R$.

We recall the definition of decomposition group and inertia group for finite extension of normal domains. Abh, page 35-37]

Definition 2.1. Let $R$ be an excellent normal domain with fraction field $K, L$ a finite $G$-Galois extension of $K$ and $S$ the integral closure of $R$ in $L$. Let $Q \subset S$ be a prime ideal of $S$ and $P=Q \cap R$.

(1) Define the decomposition group $D(Q)=\{\sigma \in G: \sigma(Q) \subset Q\}$

(2) Define the inertia group $I(Q)=\{\sigma \in G: \sigma(\alpha)-\alpha \in Q$ for all $\alpha \in S\}$. Note that $I(Q) \subset D(Q)$.

The following result is well known but a precise reference is difficult to find in the literature.

Proposition 2.2. Let $R$ be an excellent normal domain with fraction field $K, L$ a finite separable extension of $K$ and $S$ the integral closure of $R$ in $L$. Let $Q \subset S$ be a prime ideal of $S$ and $P=Q \cap R$. Let $\widehat{K}_{P}=\mathrm{QF}\left(\widehat{R}_{P}\right), \widehat{L}_{Q}=\mathrm{QF}\left(\widehat{S}_{Q}\right)$, $\widehat{K}^{P}=\mathrm{QF}\left(\widehat{R}^{P}\right)$ and $\widehat{L}^{Q}=\mathrm{QF}\left(\widehat{L}^{Q}\right)$. Then the following holds:

(1) The field extensions $\widehat{L}_{Q} / \widehat{K}_{P}$ and $\widehat{L}^{Q} / \widehat{K}^{P}$ are finite separable.

(2) If $L / K$ is Galois then so are $\widehat{L}_{Q} / \widehat{K}_{P}$ and $\widehat{L}^{Q} / \widehat{K}^{P}$. 
(3) If $G=\operatorname{Gal}(L / K)$ then $\operatorname{Gal}\left(\widehat{L}_{Q} / \widehat{K}_{P}\right)$ is isomorphic to the subgroup $D(Q)$ and $\operatorname{Gal}\left(\widehat{L}_{Q} / \widehat{K}_{P}\right) \leq \operatorname{Gal}\left(\widehat{L}^{Q} / K^{P}\right)$.

(4) Moreover, if $Q^{\prime}$ is another prime ideal of $S$ lying above $P$ then $D(Q)$ and $D\left(Q^{\prime}\right)$ are conjugates in $G$.

Proof. Since $R_{P}$ and $S_{Q}$ are excellent normal local domains, $\widehat{R}_{P}$ and $\widehat{S}_{Q}$ are also complete local normal domains containing $R_{P}$ and $S_{Q}$ respectively (EGA $4_{2}$, 7.8.3(v)]). Also note that $\widehat{R}^{P} \subset \widehat{R}_{P}$ and $\widehat{S}^{Q} \subset \widehat{S}_{Q}$. Hence $L, \widehat{K}^{P}$ and $\widehat{K}_{P}$ are subfields of $\widehat{L}_{Q}, \widehat{L}^{Q}=\widehat{K}^{P} L$ and $\widehat{L}_{Q}=\widehat{K}_{P} L$. So $\widehat{L}_{Q} / \widehat{K}_{P}$ is separable because $L / K$ is separable and $K \subset \widehat{K}_{P}$. The same proof says if $L / K$ is a normal extension then so is $\widehat{L}_{Q} / \widehat{K}_{P}$. Hence if $L / K$ is Galois then so is $\widehat{L}_{Q} / \widehat{K}_{P}$.

By definition of $D(Q)$, if $a \in Q^{n}$ then $g a \in Q^{n}$ for every $g \in D(Q)$. Hence given a Cauchy sequence $\left(\alpha_{i}\right)_{i \in \mathbb{N}} \in L$ and $g \in D(Q)$ then sequence $\left(g \alpha_{i}\right)_{i \in \mathbb{N}}$ is also a Cauchy sequence. In particular $D(Q)$ acts on $\widehat{L}_{Q}$. Also it fixes $\widehat{K}_{P}$. So $D(Q) \hookrightarrow \operatorname{Gal}\left(\widehat{L}_{Q} / \widehat{K}_{P}\right)$ is a monomorphism.

Let $\sigma \in \operatorname{Gal}\left(\widehat{L}_{Q} / \widehat{K}_{P}\right)$. Note that $L / K$ is Galois and $\sigma_{\mid K}=i d_{K}$. Hence $\sigma(L)=L$. Since $\widehat{S}_{Q}$ is the integral closure of $\widehat{R}_{P}$ in $\widehat{L}_{Q}, \sigma\left(\widehat{S}_{Q}\right)=\widehat{S}_{Q}$. But $S_{Q}=\widehat{S}_{Q} \cap L$, so $\sigma\left(S_{Q}\right)=S_{Q}$. Since $\sigma$ is a ring automorphism of a local ring, $\sigma(Q)=Q$. In particular $\sigma_{\mid L} \in D(Q)$. Hence $D(Q) \cong \operatorname{Gal}\left(\widehat{L}_{Q} / \widehat{K}_{P}\right)$.

To see the last statement, let $Q^{\prime}$ be another prime ideal of $S$ lying above $P$. We have that $S_{Q^{\prime}} \subset \widehat{S}_{Q^{\prime}}$ and hence $L \subset \widehat{L}_{Q^{\prime}}$. Let $M$ and $M^{\prime}$ be the algebraic closure of $\widehat{L}_{Q}$ and $\widehat{L}_{Q^{\prime}}$ then both $M$ and $M^{\prime}$ are algebraic closure of $\widehat{K}_{P}$. So there exists a $\widehat{K}_{P}$-isomorphism $\phi: M \rightarrow M^{\prime}$. Since $L / K$ is a Galois extension and $K \subset \widehat{K}_{P}$, $\phi(L)=L$. Note that $\widehat{L}_{Q}$ and $\widehat{L}_{Q^{\prime}}$ are splitting fields of the polynomial $f(Z)$ over $\widehat{K}_{P}$, hence $\phi\left(\widehat{L}_{Q}\right)=\widehat{L}_{Q^{\prime}}$. Since $\widehat{S}_{Q}$ and $\widehat{S}_{Q^{\prime}}$ are integral closures of $\widehat{R}_{P}$ in $\widehat{L}_{Q}$ and $\widehat{L}_{Q^{\prime}}$ respectively, $\phi\left(\widehat{S}_{Q}\right)=\widehat{S}_{Q^{\prime}}$. But $S_{Q}=\widehat{S}_{Q} \cap L$, so $\phi\left(S_{Q}\right)=S_{Q^{\prime}}$. Hence $\phi \in \operatorname{Gal}(L / K)$ and $\phi(Q)=Q^{\prime}$. In particular $\phi^{-1} D\left(Q^{\prime}\right) \phi=D(Q)$.

Proposition 2.3. Let $R$ be an excellent normal domain with fraction field $K$, $L$ a finite separable extension of $K$ and $S$ the integral closure of $R$ in $L$. Let $Q_{2} \subsetneq Q_{1} \subset S$ be prime ideals of $S$ and $P_{i}=Q_{i} \cap R$. Then $\widehat{K}^{P_{2}} \subset \widehat{K}^{P_{1}}$ and $\widehat{L}^{Q_{1}}=L \widehat{K}^{P_{1}}=\widehat{L}^{Q_{2}} \widehat{K}^{P_{1}}$.

Proof. Note that $\widehat{R}^{P_{2}}$, the $P_{2}$-adic completion of $R$, contains $R$ since $R$ is noetherian and hence $P_{2}$-adically separated. Note that $P_{2}$-adic completion of $P_{1}$ is $P_{1} \widehat{R}^{P_{2}}$ is a prime ideal of $\widehat{R}^{P_{2}}$ and $\widehat{R}^{P_{1}}$ is also the $P_{1} \widehat{R}^{P_{2}}$-adic completion of $\widehat{R}^{P_{2}}$. Hence $\widehat{R}^{P_{2}} \subset$ $\widehat{R}^{P_{1}}$. Hence $\widehat{K}^{P_{2}} \subset \widehat{K}^{P_{1}}$. Similarly $\widehat{L}^{Q_{2}} \subset \widehat{L}^{Q_{1}}$. Since $S$ is a finite $R$-module, $\widehat{S}^{Q_{1}}$ is a finite $\widehat{R}^{P_{1}}$-module with the same generators. Hence $\widehat{L}^{Q_{1}}=L \widehat{K}^{P_{1}}=\widehat{L}^{Q_{2}} \widehat{K}^{P_{1}}$.

Remark 2.4. Let $Q_{2} \subsetneq Q_{1} \subset S$ be prime ideals of $S$ and $P_{i}=Q_{i} \cap R$. If $\sigma \in I\left(Q_{2}\right)$ then $\sigma \alpha-\alpha \in Q_{2} \subset Q_{1}$ for all $\alpha \in R$. Hence $I\left(Q_{2}\right) \subset I\left(Q_{1}\right)$. (Also see Abh, Proposition 1.50])

Remark 2.5. Let $Q \subset S$ be a prime ideal of $S$ and $P=Q \cap R$. Then $I(Q)$ is the kernel of the natural epimorphism from $\phi: D(Q) \rightarrow \operatorname{Aut}\left(k_{Q} / k_{P}\right)$ where $k_{Q}=\mathrm{QF}(S / Q), k_{P}=\mathrm{QF}(R / P)$ and for $g \in D(Q), a \in S_{Q}$ and its image $\bar{a} \in k_{Q}$, $\phi(g)(\bar{a})=\overline{g a}$.

The following is a consequence of [Abh, Theorem 1.47 and 1.48] but we include a direct proof for brevity and convenience of the reader. 
Proposition 2.6. Let $(R, m)$ be a complete local normal domain and $(S, n)$ be the integral closure $R$ in a finite Galois extension of $\mathrm{QF}(R)$. Then $I(n)$ is trivial iff $S / n$ is a finite separable extension of $R / m$ and $m S=n$.

Proof. Let $k_{n}=S / n, k_{m}=R / m, L=\mathrm{QF}(S)$ and $K=\mathrm{QF}(R)$. Since $L / K$ is Galois, the field extension $k_{n} / k_{m}$ is normal. If $I(n)$ is trivial then $\operatorname{Gal}(L / K)=$ $D(n) \cong \operatorname{Aut}\left(k_{n} / k_{m}\right)$. Let $f$ be the cardinality of this group. Since $S$ is a torsion free $R$-module, the vector space dimension of $S \otimes_{R} k_{m}$ is at most $[L: K]$. Hence $\operatorname{dim}_{k_{m}}(S / m S) \leq f$. Since $m S \subset n$,

$$
f=\left|\operatorname{Aut}\left(k_{m} / k_{n}\right)\right| \leq \operatorname{dim}_{k_{m}}(S / n) \leq \operatorname{dim}_{k_{m}}(S / m S) \leq f .
$$

Hence $k_{m} / k_{n}$ is Galois extension and $m S=n$.

Conversely, by Cohen structure theorem $S=S_{0}\left[\left[y_{1}, \ldots, y_{s}\right]\right]$ and the subring $R=R_{0}\left[\left[x_{1}, \ldots, x_{r}\right]\right]$ where $R_{0}$ and $S_{0}$ are coefficient rings and $R_{0} \subset S_{0}$. Since $m S=n, S=S_{0}\left[\left[x_{1}, \ldots, x_{r}\right]\right]$. If $R_{0}$ is a field then $R_{0}=k_{m}$ and $S_{0}=k_{n}$. So $S=$ $R \otimes_{k_{m}} k_{n}$ and hence $S$ is a free $R$-module of rank $\left[k_{n}: k_{m}\right]$. So $|D(n)|=[L: K]=$ $\left[k_{n}: k_{m}\right]=\left|\operatorname{Gal}\left(k_{n} / k_{m}\right)\right|$. So the natural epimorphism from $D(n) \rightarrow \operatorname{Gal}\left(k_{n} / k_{m}\right)$ is an isomorphism. Hence $I(n)$ is trivial.

Suppose $R_{0}$ is not a field. Since $R$ and $S$ are integral domains $R_{0}$ and $S_{0}$ are characteristic zero dvrs with residue fields $K_{m}$ and $k_{n}$ are of characteristic $p>0$. Also by Cohen structure theorem, $p R_{0}$ and $p S_{0}$ are maximal ideals of $R_{0}$ and $S_{0}$ respectively. Hence $S_{0}$ is finite étale extension of $R_{0}$. Since $R_{0}$ is a dvr, $S_{0}$ is free $R_{0}$-module. Hence $S$ is a free $R$-module of $\operatorname{rank}[L: K]$. This similarly implies that $I(n)$ is the trivial group.

Definition 2.7. Let $R$ be an excellent normal domain with fraction field $K$ and $L / K$ be a finite separable extension. Let $S$ be the integral closure of $R$ in $L$, prime ideal $Q$ of $S$ and $P=Q \cap R$. The prime ideal $Q$ is said to be unramified in the extension $S / R$ or $L / K$ if $P S_{Q}=Q S_{Q}$ and $S_{Q} / Q S_{Q}$ is a finite separable extension of $R_{P} / P R_{P}$. In this scenario we will also say $S_{Q} / R_{P}$ is an unramified extension. If every $Q$ lying over $P$ is unramified then $P$ (or $R_{P}$ ) is said to be non-branched in $L$ otherwise $P\left(\right.$ or $\left.R_{P}\right)$ is said to be a branched in $L$.

Remark 2.8. By flatness of localization and completion $S_{Q} / R_{P}$ is unramified is equivalent to $\widehat{S}_{Q} / \widehat{R}_{P}$ is unramified. In the complete local ring set-up if the ring is understood from the context we may simply say $\mathrm{QF}\left(\widehat{S}_{Q}\right) / \mathrm{QF}\left(\widehat{R}_{P}\right)$ is unramified to mean $\widehat{R}_{P}$ is unramified in the field extension $\mathrm{QF}\left(\widehat{S}_{Q}\right) / \mathrm{QF}\left(\widehat{R}_{P}\right)$. By the Proposition 2.6 when $L / K$ is a Galois extension then $I(Q)$ is trivial iff then $Q$ is unramified in $L / K$.

The following is a generalization of [Kum, Lemma 3.1] to higher dimension.

Lemma 2.9. Let $R$ be a normal local excellent domain and $K$ be the quotient field of $R$. Let $L$ and $M$ be finite separable extensions of $K$ and $\Omega=L M$ their compositum. Let $A_{s}$ be the integral closure $R$ in $\Omega$ and $A$ be the localization of $A$ at a maximal ideal of $A_{s}$. Then $S=A \cap L$ and $T=A \cap M$ are normal local excellent domains. Let $\widehat{K}, \widehat{L}, \widehat{M}$ and $\widehat{\Omega}$ be the quotient field of the complete local rings $\widehat{R}, \widehat{S}$, $\widehat{T}$ and $\widehat{A}$ respectively. Here all fields are viewed as subfields of an algebraic closure of $\widehat{K}$. The following holds:

(1) If $A / m_{A}$ is compositum of $S / m_{S}$ and $T / m_{T}$ then $\widehat{\Omega}=\widehat{L} \widehat{M}$. 
(2) If $A / m_{A}$ is a separable extension of $S / m_{S}$ then $\widehat{\Omega}$ is an unramified extension of $\widehat{L} \widehat{M}$.

Proof. Note that $A_{s}$ is a semilocal ring and a finite $R$-module (since excellent rings are Nagata). Let $m_{R}$ be the maximal ideal of $R$. Note that $\widehat{A}$ is a homomorphic image of $\widehat{A}_{s}$, the $m_{R}$-adic completion of $A_{s}$. So $\widehat{A}_{s}$ and hence $\widehat{A}$ are finite $\widehat{R}$ modules.

Note that $S$ and $T$ are also a localization at maximal ideals of the integral closures of $R$ in $L$ and $M$ (respectively) dominated by $A$. Hence $\widehat{S}$ and and $\widehat{T}$ are finite $\widehat{R}$-modules. By definition $A, S, T$ are normal local domains and they are excellent because they are localization of a finite type ring over an excellent ring.

Note that $\widehat{L}$ and $\widehat{M}$ are contained in $\widehat{\Omega}$. So $\widehat{L} \widehat{M} \subset \widehat{\Omega}$. If $A / m_{A}$ is the compositum of $S / m_{S}$ and $T / m_{T}$ then there exist $\alpha_{1}, \ldots, \alpha_{l} \in T$ such that $A / m_{A}=$ $\left(S / m_{S}\right)\left[\bar{\alpha}_{1}, \ldots, \bar{\alpha}_{l}\right]$. Let $\widehat{S}_{1}$ be the integral closure of $\widehat{S}\left[\alpha_{1}, \ldots, \alpha_{l}\right]$ in the fraction field. Then $\widehat{L} \subset \mathrm{QF}\left(\widehat{S}_{1}\right) \subset \widehat{L} M$. Also $\widehat{S}_{1} / m_{\widehat{S}_{1}}=A / m_{A}$ and $\widehat{S}_{1}$ is a normal complete local domain between $\widehat{S}$ and $\widehat{A}$. So by replacing $\widehat{S}$ by $\widehat{S}_{1}$, we may assume $A / m_{A}=S / m_{S}$.

Let $m_{A}=\left(a_{1}, \ldots, a_{n}\right)$ for $a_{i} \in A \subset \widehat{A}$. Then $\widehat{S}\left[a_{1}, \ldots, a_{n}\right]$ is a finite $\widehat{S}$-module because $\widehat{A}$ is finite over $\widehat{R}$ and $\widehat{R} \subset \widehat{S}$. Hence $\widehat{S}\left[a_{1}, \ldots, a_{n}\right]$ is a complete local ring by [Coh, Theorem 7] with maximal ideal $m$ (say). Also $\widehat{S} \subset \widehat{S}\left[a_{1}, \ldots, a_{n}\right] \subset \widehat{A}$ are extensions of local rings with $\widehat{S} / m_{\widehat{S}}=\widehat{A} / m_{\widehat{A}}$. Hence the $\widehat{S}\left[a_{1}, \ldots, a_{n}\right]$ and $\widehat{A}$ have the same residue field. Also $m_{\widehat{A}}=m_{\widehat{S}} \widehat{A}$ since $m_{\widehat{A}} \supset m_{\widehat{A}} \supset m_{A}$ and $m_{A} \widehat{A}=m_{\widehat{A}}$. Hence by [Coh, Corollary to Theorem 8], $\widehat{S}\left[a_{1}, \ldots, a_{n}\right]=\widehat{A}$. So $\widehat{\Omega}=\widehat{L}\left[a_{1} \ldots, a_{n}\right] \subset$ $\widehat{L} M \subset \widehat{L} \widehat{M}$. This completes the proof of the first part.

Let $\widehat{B}$ be the integral closure of $\widehat{S}$ in $\widehat{L} \widehat{M}$. Since $\widehat{\Omega} \supset \widehat{L} \widehat{M}, \widehat{A} \supset \widehat{B}$ and $\widehat{A}$ is a finite $\widehat{B}$-module (as $\widehat{B}$ contains $\widehat{S}$ ). So $m_{\widehat{B}}=m_{\widehat{A}} \cap \widehat{B}$. Note that $a_{1}, \ldots a_{n} \in m_{\widehat{B}}$ since $a_{1}, \ldots, a_{n} \in m_{A} \subset L M \subset \widehat{L} \widehat{M}$. So $m_{\widehat{B}} \widehat{A}=m_{\widehat{A}}$. Moreover $\widehat{S} / m_{\widehat{S}} \subset \widehat{B} / m_{\widehat{B}} \subset$ $\widehat{A} / m_{\widehat{A}}$ and the hypothesis for (2) implies $\widehat{A} / m_{\widehat{A}}$ is a separable extension of $\widehat{B} / m_{\widehat{B}}$. Hence by Proposition 2.6 $\widehat{A}$ is an unramified extension of $\widehat{B}$, i.e. $\widehat{\Omega}$ is an unramified extension of $\widehat{L} \widehat{M}$.

Corollary 2.10. Let the notation be as in the above lemma. If $\widehat{L} \subset \widehat{M}$ then $A / T$ is an unramified extension.

Proof. Since $\Omega / M$ is a finite extension, so is $\widehat{\Omega} / \widehat{M}$. Hence $\widehat{A}$ is a finite $\widehat{T}$-module. The above lemma and the hypothesis implies that $\widehat{\Omega}=\widehat{M}$. So $\widehat{A}=\widehat{T}$, i.e. $A / T$ is unramified.

\section{BRANCH DATA}

For an excellent normal scheme $X$ and $x \in X$, let $\mathcal{K}_{X, x}$ be the fraction field of $\widehat{\mathcal{O}}_{X, x}$. Let $\bar{x}$ denote the closure of $\{x\}$ in $X$. Let $\widehat{X}_{\bar{x}}$ be the formal scheme obtained by the completion of $X$ along $\bar{x}$. For an open affine connected subset $U$ of $X$ containing $x$, let $\mathcal{K}_{X}^{x}(U)=\mathrm{QF}\left(\widehat{R}^{I}\right)$ where the coordinate ring of $U$ is $R$ and $I$ is the (prime) ideal of $R$ defining $x$. Note that $\mathcal{K}_{X}^{x}(U)=\mathrm{QF}\left(\mathcal{O}_{\widehat{X}_{\bar{x}}}(U \cap \bar{x})\right)$.

Proposition 3.1. In the above setup, for $x \in X$ and $U \subset V$ be two affine open connected neighbourhood of $x$ in $X, \mathcal{O}_{\widehat{X}_{\bar{x}}}(U \cap \bar{x}) \supset \mathcal{O}_{\widehat{X}_{\bar{x}}}(V \cap \bar{x})$ and the completion 
of $\mathcal{O}_{\widehat{X}_{\bar{x}, x}}$ at the maximal ideal is $\widehat{\mathcal{O}}_{X, x}$. In particular we have the inclusion of fields $\mathcal{K}_{X}^{x}(V) \subset \mathcal{K}_{X}^{x}(U) \subset \mathcal{K}_{X, x}$.

Proof. The first statement follows from ([EGA 1, Proposition 10.1.4]) and noting that $U$ and $V$ are affine integral schemes. Since $X$ is normal excellent schemes, all the three rings are integral domains (EGA 42, 7.8.3]) and hence admit fraction fields.

Proposition 3.2. In the above setup let $L / \mathcal{K}_{X}^{x}(U)$ be a finite Galois extension and $R=\mathcal{O}_{\widehat{X}_{\bar{x}}}(U \cap \bar{x})$. Let $S$ be the integral closure of $R$ in L. Let $y \in \operatorname{Spec}(S)$ be a point lying above $x \in \operatorname{Spec}(R)$ and $I$ and $J$ be the ideals defining $x \in \operatorname{Spec}(R)$ and $y \in \operatorname{Spec}(S)$ respectively. The morphism $\operatorname{Spec}(S) \rightarrow \operatorname{Spec}(R)$ is unramified at $x$ iff $\widehat{S}_{J} / \widehat{R}_{I}$ is an unramified extension of complete local rings. In particular under the assumption that $L / \mathcal{K}_{X}^{x}(U)$ is Galois, $x$ is not branched in the morphism $\operatorname{Spec}(S) \rightarrow \operatorname{Spec}(R)$ iff $\widehat{\mathcal{O}}_{X, x}$ is unbranched in the extension $L \mathcal{K}_{X, x} / \mathcal{K}_{X, x}$.

Proof. By definition the morphism $\operatorname{Spec}(S) \rightarrow \operatorname{Spec}(R)$ is unramified at $x$ iff $I S_{J}=$ $J S_{J}$ and $S_{J} / J S_{J}$ is a separable extension of $R_{I} / I R_{I}$. But passing to the completion does not change the residue fields and the identity $I \widehat{S}_{J}=J \widehat{S}_{J}$ also holds. Hence $\widehat{S}_{J} / \widehat{R}_{I}$ is unramified extension. If $L / \mathcal{K}_{X}^{x}(U)$ is Galois then $x$ is not branched in the morphism $\operatorname{Spec}(S) \rightarrow \operatorname{Spec}(R)$ iff $\widehat{S}_{J} / \widehat{R}_{I}$ is an unramified extension (as all the points lying above $x$ are conjugates). But $\widehat{R}_{I}=\widehat{\mathcal{O}}_{X, x}$ and $\mathrm{QF}\left(\widehat{S}_{J}\right)=L \mathcal{K}_{X, x}$.

Definition 3.3. A quasi-branch data on an excellent normal scheme $X$ is a function $P$ which to every point $x \in X$ of codimension at least one and an open affine connected $U \subset X$ containing $x$, assigns a finite Galois extension $P(x, U) / \mathcal{K}_{X}^{x}(U)$ in some fixed algebraic closure of $\mathcal{K}_{X}^{x}(U)$ such that $P\left(x_{1}, U\right)=P\left(x_{2}, U\right) \mathcal{K}_{X}^{x_{1}}(U)$ whenever $x_{1} \in \overline{\left\{x_{2}\right\}}$ and for $x \in V \subset U \subset X$ with $V$ affine open connected $P(x, V)=P(x, U) \mathcal{K}_{X}^{x}(V)$.

We let $P(x)=P(x, U) \mathcal{K}_{X, x}$. The branch locus of $P, \operatorname{BL}(P)=\{x \in X$ : $\widehat{\mathcal{O}}_{X, x}$ is branched in $\left.P(x)\right\}$. The function $P$ will be called a branch data if $\operatorname{BL}(P)$ is a closed subset of $X$ of codimension at least one. The branch data in which all the finite extensions are trivial is called the trivial branch data and is denoted by $O$. A branch data with empty branch locus is called an essentially trivial branch data.

Note that when $x \in X$ is a closed point then $\mathcal{K}_{X}^{x}(U)$ is independent of the of the choice of $U$ and hence this definition of branch data agrees with $\overline{\mathrm{KP}}$, Definition 2.1] when $X$ is of dimension one.

Corollary 3.4. Let $X$ be a normal integral excellent scheme with function field $K$ and $L / K$ be a finite separable extension. Let $Y$ be the normalization of $X$ in $L$ and $f: Y \rightarrow X$ be the corresponding morphism. Let $y_{1}, y_{2}$ be points of $Y$ such that $y_{1} \in \overline{\left\{y_{2}\right\}}$ and let $x_{i}=f\left(y_{i}\right)$ for $i=1,2$. Let $U$ be an open affine connected neighbourhood of $x$ and $V=f^{-1}(U)$. Then $\mathcal{K}_{X}^{x_{2}}(U) \subset \mathcal{K}_{X}^{x_{1}}(U)$ and $\mathcal{K}_{Y}^{y_{1}}(V)=L \mathcal{K}_{X}^{x_{1}}(U)=\mathcal{K}_{Y}^{y_{2}}(V) K_{X}^{x_{1}}(U)$.

Proof. This is a translation of Proposition 2.3 .

Some of the notions and properties of branch data ([KP, 2.3,2.4,2.5]) which hold for curves extend to higher dimension as shown below. 
Proposition 3.5. Let $X$ be a normal integral excellent scheme with function field $K$ and $L / K$ be a finite Galois extension. Let $Y$ be the normalization of $X$ in $L$ and $f: Y \rightarrow X$ be the corresponding morphism. For $x \in X$ of codimension at least one and $U$ an open affine connected neighbourhood of $x$, let $y \in Y$ be such that $f(y)=x$ and $V=f^{-1}(U)$, define $B_{f}(x, U)=\mathcal{K}_{Y}^{y}(V)$. Then $B_{f}$ is a branch data on $X$. Moreover if $X$ is nonsingular then $\mathrm{BL}\left(B_{f}\right)$ is either empty or pure of co-dimension one.

Proof. Since $L / K$ is a Galois extension, all points in $f^{-1}(x)$ for any point $x \in$ $X$ are conjugates and $\mathcal{K}_{Y}^{y}(V) / \mathcal{K}_{X}^{x}(U)$ are isomorphic Galois extensions for every $y \in f^{-1}(x)$. Hence $B_{f}(x, U) / \mathcal{K}_{X}^{x}(U)$ is a Galois extension and it is independent of the choice of $y \in Y$ lying above $x$. That $B_{f}$ is a quasi branch data follows from Corollary 3.4 and Proposition 3.1 .

The ramification locus of $f$ is a proper closed subset of $Y$ and $f$ is a proper morphism. Hence the branch locus of $f$ in $X$ is a proper closed subset. Hence $B_{f}$ is a branch data. The moreover part follows from Zariski's purity of branch locus.

Definition 3.6. Let $P$ and $Q$ be quasi-branch data on $X$. Define their intersection $(P \cap Q)(x, U)=P(x, U) \cap Q(x, U)$ and their compositum $(P Q)(x, U)=$ $P(x, U) Q(x, U)$ for all $x \in X$ of codimension at least one and $U$ any affine open neighbourhood of $x$.

Lemma 3.7. Compositum of two (quasi-)branch data is a (quasi-)branch data.

Proof. Note that the compositum of two Galois extensions are Galois. So it follows that if $P$ and $Q$ are quasi-branch data then so is $P Q$.

Moreover $\mathrm{BL}(P Q)=\mathrm{BL}(P) \cup \mathrm{BL}(Q)$. So if $P$ and $Q$ are branch data then so is $P Q$.

Proposition 3.8. Let $P$ be a branch data on $X$ and $f: Y \rightarrow X$ be a quasi-finite dominant separable morphism between normal excellent schemes. For $y \in Y$, let $U$ be an affine open connected neighbourhood of $f(y)$ so that $P(f(y), U)$ is a finite Galois extension of $\mathcal{K}_{X}^{f(y)}(U)$. Let $V$ be an affine open connected neighbourhood of $y$ contained in $f^{-1}(U)$ and $Q(y, V)=\mathcal{K}_{Y}^{y}(V) P(f(y), U)$. Then $Q$ is a branch data on $Y$ and will be denoted by $f^{*} P$.

Proof. Let $y \in Y$ and $x=f(y)$ and $U$ be an affine open connected neighbourhood of $x$ for which $P(x, U) / \mathcal{K}_{X}^{x}(U)$ is a Galois extension. Since $\mathcal{K}_{X}^{x}(U) \subset \mathcal{K}_{Y}^{y}(V)$ for any $V$ affine open subset of $f^{-1}(U)$ containing $y, Q(y, V) / \mathcal{K}_{Y}^{y}(V)$ is a Galois extension. Also if $y_{1} \in \overline{\left\{y_{2}\right\}}$ then $f\left(y_{1}\right) \in \overline{\left\{f\left(y_{2}\right)\right\}}$. Hence for some affine open connected neighbourhood $U$ of $f\left(y_{1}\right), P\left(f\left(y_{1}\right), U\right)=P\left(f\left(y_{2}\right), U\right) \mathcal{K}_{X}^{f\left(y_{1}\right)}(U)$. So for $V$ an affine open connected subset of $f^{-1}(U)$ containing $y_{1}, Q\left(y_{1}, V\right)=\mathcal{K}_{Y}^{y_{1}}(V) P\left(f\left(y_{1}\right), U\right)=$ $\mathcal{K}_{Y}^{y_{1}}(V) P\left(f\left(y_{2}\right), U\right) \mathcal{K}_{X}^{f\left(y_{1}\right)}(U)=\mathcal{K}_{Y}^{y_{1}}(V) P\left(f\left(y_{2}\right), U\right)$.

By Proposition $2.3 \mathcal{K}_{Y}^{y_{2}}(V) \subset \mathcal{K}_{Y}^{y_{1}}(V)$ so $Q\left(y_{1}, V\right)=\mathcal{K}_{Y}^{y_{1}}(V) P\left(f\left(y_{2}\right), U\right) \mathcal{K}_{Y}^{y_{2}}(V)$. Hence $Q\left(y_{1}, V\right)=Q\left(y_{2}, V\right) \mathcal{K}_{Y}^{y_{1}}(V)$.

Finally by Remark 2.4 if $y \in \operatorname{BL}(Q)$ then every point in $\overline{\{y\}}$ is in $\operatorname{BL}(Q)$. Hence $\mathrm{BL}(Q)$ is a closed subset of $f^{-1}(\mathrm{BL}(P))$. Hence $Q$ is a branch data.

Definition 3.9. Let $P$ and $Q$ be two branch data on a normal excellent scheme $X$. We say $P$ is less than or equal to $Q$ (and write $P \leq Q$ ) if for all points $x \in X$ of codimension at least one and an affine open connected neighbourhood $U$ of $x$, 
$P(x, U) \subset Q(x, U)$. In particular $P(x) \subset Q(x)$ or all points $x \in X$ of codimension at least one.

Notation. Let $x \in X$ be a point and $\mathcal{K}_{X, x} \subset L_{1} \subset L_{2}$ be finite field extension. Then we say the extension $L_{2} / L_{1}$ is ramified (or unramified) if integral closure of $\widehat{\mathcal{O}}_{X, x}$ in $L_{2}$ is ramified (or unramified) extension of integral closure of $\widehat{\mathcal{O}}_{X, x}$ in $L_{1}$.

\section{Formal ORBIFOLDS}

Now we are ready to extend the definition of formal orbifolds for curves and morphisms between them in $[\mathrm{KP}$, Definition 2.1(3), 2.6] to arbitrary dimension and many of the basic properties also extend. Another difference from $\mathrm{KP}$ in the treatment is that we allow morphisms to be quasi finite (as opposed to finite morphisms).

Definition 4.1. A formal orbifold is a pair $(X, P)$ where $X$ is a finite type reduced normal scheme over a perfect field $k$ or $\operatorname{Spec}(\mathbb{Z})$ and $P$ is a branch data on $X$.

(1) An admissible morphism of formal orbifolds $f:(Y, Q) \rightarrow(X, P)$ is a quasifinite dominant separable morphism $f: Y \rightarrow X$ of normal excellent schemes such that $Q\left(y, f^{-1}(U)\right) \supset P(f(y), U)$ for all points $y \in Y$ of codimesion at least one and for some $U$ affine open connected neighbourhood of $f(y)$ with $f^{-1}(U)$ affine.

(2) An admissible morphism $f$ is called flat morphism if $f: Y \rightarrow X$ is flat away from $f^{-1}(\mathrm{BL}(P))$.

(3) An admissible morphism $f$ is said to be unramified or étale at $y$ if the extension $Q(y) / P(f(y))$ is unramified and $f$ is called étale morphism if $f$ is unramified for all $y \in Y$.

(4) An admissible morphism is called a covering morphism if it is also proper (and hence finite).

(5) A covering morphism $f$ is called an étale cover if $f$ is an étale covering morphism.

Proposition 4.2. Composition of admissible (respectively étale) morphisms is admissible (respectively étale).

Proof. Let $f:(Y, Q) \rightarrow(X, P)$ and $g:(Z, B) \rightarrow(Y, Q)$ be admissible morphism of formal orbifolds. Then $f \circ g$ is a quasi-finite dominant separable morphism as these properties are preserved under composition. Since $f$ and $g$ are admissible, for any $z \in Z$ there exists $U$ affine open connected neighbourhood of $g(z)$ such that $g^{-1}(U)$ is affine and $B\left(z, g^{-1}(U)\right) \supset Q(g(z), U)$. Similarly there exists $V$ affine open connected neighbourhood of $f \circ g(z)$ such that $f^{-1}(V)$ is affine and $Q\left(g(z), f^{-1}(V)\right) \supset P(f \circ g(z), V)$. Shrinking $V$ if necessary we may assume $f^{-1}(V)$ is an affine open subset of $U$ and $W=g^{-1}\left(f^{-1}(V)\right.$ is affine. Now $B(z, W)=B\left(z, g^{-1}(U)\right) \mathcal{K}_{Z}^{z}(W) \supset Q(g(z), U) \mathcal{K}_{Y}^{g(z)}\left(f^{-1}(V)\right)=Q\left(g(z), f^{-1}(V)\right)$ and hence $B(z, W) \supset P(f \circ g(z), V)$. Hence $f \circ g$ is an admissible morphism.

Note that being unramified is preserved under composition, hence if $f$ and $g$ are unramified at all points then so is $f \circ g$.

The following is a known result but perhaps not written down explicitly. 
Proposition 4.3. Let $f: Y \rightarrow X$ be a quasi-finite dominant morphism between normal schemes which is unramified at $y \in Y$. Then $f$ is flat at $y$.

Proof. Let $y \in Y$ and $x=f(y)$. Let $A$ be the strict henselianization of $\mathcal{O}_{X, x}$ then $A$ is a normal domain faithfully flat over $\mathcal{O}_{X, x}$. Now $C=A \otimes_{\mathcal{O}_{X, x}} \mathcal{O}_{Y, y}$ is a finite unramified extension of $A$ and $A$ is a strictly henselian integral domain, hence $C$ is a free $A$-module and hence flat. Since $A / \mathcal{O}_{X, x}$ is faithfully flat, the original extension $\mathcal{O}_{Y, y} / \mathcal{O}_{X, x}$ is flat.

Corollary 4.4. Let $f:(Y, Q) \rightarrow(X, P)$ be an étale morphism. Then $f$ is flat away from $f^{-1}(\mathrm{BL}(P))$.

Proof. Let $y \in Y$ be such that $y \notin f^{-1}(\operatorname{BL}(P))$ and let $x=f(y)$. Then $P(x) / \mathcal{K}_{X, x}$ is unramified. Since $f$ is étale $Q(y) / P(x)$ is unramified. Hence $Q(y) / \mathcal{K}_{X, x}$ is unramified which implies $\mathcal{K}_{Y, y} / \mathcal{K}_{X, x}$ is unramified. Hence $f: Y \rightarrow X$ is unramified at $y$ and $X$ is normal. This implies $f$ is flat at $y$ by the above proposition.

Proposition 4.5. Let $f:(Y, Q) \rightarrow(X, P)$ and $g:(Z, B) \rightarrow(Y, Q)$ be flat morphisms of formal orbifolds such that $Q / f^{*} P$ or equivalently id $:(Y, Q) \rightarrow\left(Y, f^{*} P\right)$ is unramified. Then $f \circ g$ is flat.

Proof. Note that $\mathrm{BL}(Q)=\mathrm{BL}\left(f^{*} P\right)$ and by Proposition 3.8 $\mathrm{BL}\left(f^{*} P\right) \subset f^{-1}(\mathrm{BL}(P))$. Hence $(f \circ g)^{-1}(\mathrm{BL}(P))=g^{-1}\left(f^{-1}(\mathrm{BL}(P))\right) \supset g^{-1}(\mathrm{BL}(Q))$. So if $z \notin(f \circ$ $g)^{-1}(\mathrm{BL}(P))$ then $z \notin g^{-1}(\mathrm{BL}(Q))$, hence $g$ is flat (as morphism of schemes) at $z$ and $g(z) \notin f^{-1}(\mathrm{BL}(P))$. Hence $f$ is flat at $g(z)$ which implies $f \circ g$ is flat at $z$.

Definition 4.6. Let $f:(Y, Q) \rightarrow(X, P)$ be a covering morphism of formal orbifolds. The ramification locus of $f$ is $\{y \in Y: Q(y) / P(f(y))$ is a ramified extension $\}$. Its image in $X$ is called the branch locus of $f$.

Lemma 4.7. Let $f:(Y, Q) \rightarrow(X, P)$ be a covering morphism of formal orbifolds then the ramification locus and the branch locus of $f:(Y, Q) \rightarrow(X, P)$ are closed.

Proof. Since $f$ is proper it is enough to show that the ramification locus is closed. Let $y \in Y$ be in the ramification locus of $f$ and $y_{0} \in \overline{\{y\}}$ be a point. Let $x=$ $f(y)$ and $x_{0}=f\left(y_{0}\right)$. Note that $x_{0}$ is in the closure of $x$. Let $U$ be an affine open connected neighbourhood of $x_{0}$ and $V=f^{-1}(U)$. Since $f$ is ramified at $y$, by Proposition $3.2 y$ is ramified in the field extension $Q(y, V) / P(x, U)$. Also $P\left(x_{0}, U\right)=P(x, U) \mathcal{K}_{X}^{x_{0}}(U)$ and $Q\left(y_{0}, V\right)=Q(y, V) \mathcal{K}_{Y}^{y_{0}}(V)$. So we have

$$
\begin{aligned}
Q(y, V) P\left(x_{0}, U\right) & =Q(y, V) \mathcal{K}_{X}^{x_{0}}(U) \\
& =Q(y, V) K_{Y}^{y}(V) K_{X}^{x_{0}}(U) \\
& =Q(y, V) K_{Y}^{y_{0}}(V) \text { (by Proposition 2.3). } \\
& =Q\left(y_{0}, V\right)
\end{aligned}
$$

Let $A_{0}$ be the normalization of $\widehat{\mathcal{O}}_{X, x_{0}}$ in $P\left(x_{0}\right)$ and $B_{0}$ be the normalization of $A_{0}$ in $Q\left(y_{0}\right)$. Let $I$ be the ideal of $\mathcal{O}_{X}(U)$ defining the point $x$ and $J$ be the ideal of $\mathcal{O}_{Y}(V)$ defining $y$. Note that $J \cap \mathcal{O}_{X}(U)=I$. Let $A$ be the normalization of ${\widehat{\mathcal{O}_{X}(U)}}^{I}$ in $P(x, U)$ and $B$ be the normalization of $A$ in $Q(y, V)$ (which is same as the normalization of $\widehat{\mathcal{O}_{Y}(V)}{ }^{J}$ ). Note that $A_{0}$ is the completion of the stalk of $\operatorname{Spec}(A)$ at a point lying above $x_{0}$ and similarly $B_{0}$. 


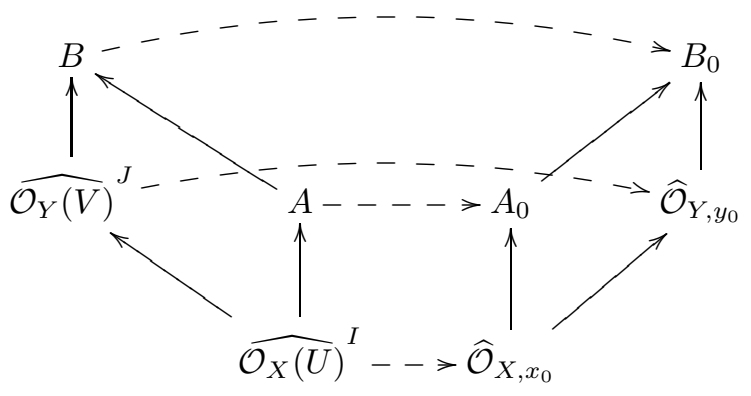

Hence there are prime ideals in $B$ denoted by $\tilde{J} \subset \tilde{J}_{0}$ which corresponds to points $y$ and $y_{0}$ of $Y$. Moreover $\tilde{J}$ and $\tilde{J}_{0}$ lie over prime ideals in $A$ which corresponds to points $x$ and $x_{0}$ of $X$ respectively. Now using Remark 2.4 the inertia group $I(\tilde{J})$ in the extension $B / A$ is a subgroup of $I\left(\tilde{J}_{0}\right)$. Since $Q(y) / P(x)$ is ramified $I(\tilde{J})$ is a nontrivial group and hence $I\left(\tilde{J}_{0}\right)$ is also a nontrivial group. This implies $\tilde{J}_{0}$ is ramified in $B / A$ and passing to the completion at $\tilde{J}_{0}$ one gets $Q\left(y_{0}\right) / P\left(y_{0}\right)$ is ramified. So $y_{0}$ is in the ramification locus of $\left.f:(Y, Q) \rightarrow X, P\right)$. Hence the ramification locus is closed.

Lemma 4.8. Let $(Y, Q)$ and $(X, P)$ be formal orbifolds. Let $f: Y \rightarrow X$ be a finite morphism such that for all codimension one point $y \in Y$ and some affine open connected neighbourhood $U$ of any $x_{0} \in \overline{\{f(y)\}}, Q\left(y, f^{-1}(U)\right) \supset P(f(y), U)$. Then $f:(Y, Q) \rightarrow(X, P)$ is a covering morphism of formal orbifolds.

Proof. Let $y_{0}$ be a point of $Y$ of codimension at least two and $y_{1}$ be a codimension one point of $Y$ such that $y_{0} \in \overline{\left\{y_{1}\right\}}$. Let $x_{i}=f\left(y_{i}\right)$ for $i=0,1$ and $U$ be an affine open connected neighbourhood of $x_{0}$ such that $Q\left(y_{1}, V\right) \supset P\left(x_{1}, U\right)$ where $V=f^{-1}(U)$. Since $P, Q$ are branch data, $Q\left(y_{0}, V\right)=Q\left(y_{1}, V\right) \mathcal{K}_{Y}^{y_{0}}(V)$ and $P\left(x_{0}, U\right)=P\left(x_{1}, U\right) \mathcal{K}_{X}^{x_{0}}(U)$. By assumption $Q\left(y_{1}, V\right) \supset P\left(x_{1}, U\right)$ and since $f$ is a finite morphism $\mathcal{K}_{Y}^{y_{0}}(V) \supset \mathcal{K}_{X}^{x_{0}}(U)$. Hence $Q\left(y_{0}, V\right) \supset P\left(x_{0}, U\right)$.

Definition 4.9. A formal orbifold $(X, P)$ is called a geometric formal orbifold if there exists an étale cover $f:(Y, Q) \rightarrow(X, P)$ where $Q$ is an essentially trivial branch data on $Y$. In this case $P$ is called a geometric branch data on $X$.

The following analogue of [KP Lemma 2.12] holds in higher dimension as well with essentially the same proof.

Proposition 4.10. Let $(X, P)$ be an integral formal orbifold with function field $K$ and $f: Y \rightarrow X$ be a finite covering where $Y$ is normal. Then for a branch data $Q$ on $Y, Q \geq f^{*} P$ iff $f:(Y, Q) \rightarrow(X, P)$ is a morphism of formal orbifolds. Moreover if $Q=f^{*} P$ then $f$ is unramified iff $\mathcal{K}_{Y, y} P(f(y)) / P(f(y))$ is an unramified extension for all $y \in Y$.

Remark 4.11. Let $(X, P)$ be a formal orbifold, $f: Y \rightarrow X$ be a cover and $Q=$ $f^{*} P$. If $y \in \mathrm{BL}(Q)$ then $Q(y) / \mathcal{K}_{Y, y}$ is ramified. But $Q(y)=P(f(y)) \mathcal{K}_{Y, y}$, hence $P(f(y)) / \mathcal{K}_{X, f(y)}$ is ramified. Hence $f(y) \in \mathrm{BL}(P)$, i.e., $\mathrm{BL}(Q) \subset f^{-1}(\mathrm{BL}(P))$

Corollary 4.12. Let $f: Y \rightarrow X$ be a finite covering of normal varieties. Then $(Y, O) \rightarrow\left(X, B_{f}\right)$ is étale.

Proof. By Proposition 3.5. $\left(X, B_{f}\right)$ is a formal orbifold. Moreover by definition of $B_{f}, B_{f}\left(f(y), f^{-1}(U)\right)=\mathcal{K}_{Y}^{y}\left(f^{-1}(U)\right)$ for all $y \in Y$ and $U$ an affine open connected 
neighbourhood of $f(y)$. Note that $f^{*} B_{f}=O$. Hence by the previous proposition $(Y, O) \rightarrow\left(X, B_{f}\right)$ is unramified at all $y \in Y$. Hence the morphism $(Y, O) \rightarrow\left(X, B_{f}\right)$ of formal orbifolds is étale.

Proposition 4.13. Let $(X, P)$ be a formal orbifold. There exists a closed subset $Y$ in $X$ of codimension at least two and an open cover $\left\{U_{i}\right\}$ of $X \backslash Y$ such that $\left(U_{i},\left.P\right|_{U_{i}}\right)$ is a geometric formal orbifold.

Proof. We may assume $X$ is connected and hence integral. Let $B_{1}, \ldots, B_{r}$ be the irreducible component of $\mathrm{BL}(P)$ of codimension one in $X$. Let $x_{1}, \ldots, x_{r}$ be the generic points of $B_{1}, \ldots, B_{r}$. Let $L_{i} / k(X)$ be a finite separable field extension such that $L_{i} \mathcal{K}_{X, x_{i}}=P\left(x_{i}\right)$. Let $f_{i}: V_{i} \rightarrow X$ be the normalization of $X$ in $L_{i}$ and $Q_{i}=f_{i}^{*} P$. By construction $f_{i}:\left(V_{i}, Q_{i}\right) \rightarrow(X, P)$ is unramified above $x_{i}$ and $\mathrm{BL}\left(Q_{i}\right)$ does not contain $x_{i}$. Let $Z_{i}$ be the branch locus of $f_{i}:\left(V_{i}, Q_{i}\right) \rightarrow(X, P)$. Let $U_{i}=X \backslash\left(f_{i}\left(\mathrm{BL}\left(Q_{i}\right)\right) \cup Z_{i}\right)$. Then $x_{i} \in U_{i}$. Let $U_{0}=X \backslash \mathrm{BL}(P)$. Note that $\left(U_{i},\left.P\right|_{U_{i}}\right)$ is a geometric formal orbifold for $i=0,1, \ldots, r$ (for $i=0, i d_{U_{0}}$ is étale and the branch data $\left.P\right|_{U_{0}}$ is essentially trivial; for other $U_{i}^{\prime}$ 's $\left.f_{i}\right|_{f_{i}^{-1}\left(U_{i}\right)}$ are étale coverings of formal orbifolds and $\left.Q_{i}\right|_{f_{i}^{-1}\left(U_{i}\right)}$ are essentially trivial branch data). Note that $\cup_{i=0}^{r} U_{i}$ is the complement of a codimension 2 subset of $X$.

Remark 4.14. A formal orbifold which has an open cover by geometric formal orbifolds can possibly be interpreted as Deligne-Mumford stacks. But viewing them as varieties with branch data is more natural and useful to study the ramification theoretic properties of the varieties. This point of view is also more concrete and elementary.

Lemma 4.15. Let $f:(Y, P) \rightarrow(X, B)$ and $g:(Z, Q) \rightarrow(X, B)$ be admissible morphisms between formal orbifolds. Let $W$ be the normalization of $Y \times{ }_{X} Z$ and $p_{Y}: W \rightarrow Y$ and $p_{Z}: W \rightarrow Z$ be the projection morphisms and let $A=p_{Y}^{*} P p_{Z}^{*} Q$. Then $A$ is a branch data on $W$. For a point $w \in W$, let $y=p_{Y}(w), z=p_{Z}(w)$ and $x=f(y)=g(z)$. If $w$ is a closed point then $A(w)$ is an unramified extension of $P(y) Q(z)$.

Proof. Since $p_{Y}$ and $p_{Z}$ are quasi-finite dominant separable morphisms $p_{Y}^{*} P$ and $p_{Z}^{*} Q$ are branch data on $W$ by Proposition 3.8. Hence $A$ being compositum of two branch data is a branch data (Lemma 3.7). For $w \in W$ a closed point, $p_{Y}^{*} P(w)=$ $P(y) K_{W, w}$ and $p_{Z}^{*} Q(w)=Q(z) K_{W, w}$. Hence $A(w)=P(y) Q(z) K_{W, w}$. As $w$ is a closed point of $W$ and $W$ is a finite type scheme over a perfect field or $\operatorname{Spec}(\mathbb{Z})$, the residue field $k(w)$ is a separable extension of $k(y)$. So by Lemma $2.9 \mathcal{K}_{W, w} / \mathcal{K}_{Y, y} \mathcal{K}_{Z, z}$ is an unramified extension. Hence by base change $A(w) / P(y) Q(z)$ is an unramified extension.

Proposition 4.16. In the above setup, $p_{Y}:(W, A) \rightarrow(Y, P), p_{Z}:(W, A) \rightarrow(Z, Q)$ are admissible morphisms and $(W, A)$ is the fiber product in the category of formal orbifolds where morphisms are admissible morphisms.

Proof. Since $f: Y \rightarrow X$ and $g: Z \rightarrow X$ are quasi finite dominant morphisms their base change $p_{Y}$ and $p_{Z}$ are also quasi finite dominant morphism. Also by definition of $A, A\left(w, p_{Y}^{-1}(U)\right) \supset P\left(p_{Y}(w), U\right)$ for some affine open connected neighbourhood $U$ of $p_{Y}(w)$. Hence $p_{Y}$ and $p_{Z}$ are morphisms of formal orbifold. 
Note that the normalized fiber product has the universal property of the fiber product in the category of normal schemes. This together with the definition of $A$ implies that $(W, A)$ is the fiber product of $f$ and $g$.

Proposition 4.17. Let $f:(Y, P) \rightarrow(X, B)$ be an étale morphism and $g:(Z, Q) \rightarrow$ $(X, B)$ is an admissible morphism. Then pullback of $g, p_{Z}:(W, A) \rightarrow(Z, Q)$ is étale where $(W, A)$ is the formal orbifold fiber product of $f$ and $g$.

Proof. By Lemma 4.7 to check whether $p_{Z}$ is étale it is enough to check that $p_{Z}$ is unramified at the closed points of $W$. Let $w \in W$ be a closed point, $z=p_{Z}(w)$, $y=p_{Y}(w)$ and $x=f(y)=g(z)$. By hypothesis $P(y) / B(x)$ is an unramified extension so $P(y) Q(z) / Q(z)$ is also unramified. Since $w$ is a closed point of $W$ by Lemma $4.15 A(w) / P(y) Q(z)$ is unramified. Hence $A(w) / Q(z)$ is unramified.

Corollary 4.18. The fiber product of two étale covering morphisms is étale.

Proof. This follows from Proposition 4.17 and Proposition 4.2

\section{5. ÉtALE FUNDAMENTAL GROUP}

Once we have the category of étale coverings of a formal orbifold. The usual technique introduced by Grothendieck in [SGA1] (and also used in [KP, Section $2.2]$ ) can be used to define the étale fundamental group of a connected formal orbifold. The results in this section are also generalization of some of the results in KP Section 2.2] on fundamental group of formal orbifold curves over algebraically closed fields to higher dimension formal orbifolds. The proofs are also along the same lines using higher dimensional analogue of the required results proved in the previous sections and some minor modifications.

Definition 5.1. Let $\mathcal{X}=(X, P)$ be a connected formal orbifold and let $\operatorname{Cov}(\mathcal{X})$ be the category of all finite étale covers of $\mathcal{X}$. Let $\tilde{\mathcal{X}}$ be the inverse limit of the inverse system $\left\{\left(Y_{i}, Q_{i}\right) \in \operatorname{Cov}(\mathcal{X})\right\}_{i \in I}$ of connected finite étale covers of $\mathcal{X}$. Given a geometric point $x$ of $X$ such that its image in $X$ is not in $\operatorname{Supp}(P)$ and $\tilde{x} \in$ $\tilde{\mathcal{X}}$ lying above $x$. The fiber functor $F_{x}$ from the category of finite étale covers $\operatorname{Cov}(\mathcal{X})$ to the category of sets is pro-representable by $\tilde{\mathcal{X}}$ via $\tilde{x}$. So the group of automorphism of $F_{x}$ is isomorphic to $\operatorname{Aut}(\tilde{\mathcal{X}} / X)$. Define the étale fundamental group of $\mathcal{X}$ to be $\operatorname{Aut}(\tilde{\mathcal{X}} / X)$ which is same as $\lim _{i \in I} \operatorname{Aut}\left(Y_{i} / X\right)$ over all connected étale covers $\left(Y_{i}, Q_{i}\right) \rightarrow \mathcal{X}$. This group will be denoted by $\pi_{1}(\mathcal{X}, x)$ or simply $\pi_{1}(\mathcal{X})$.

Proposition 5.2. Let $f: \mathcal{Y} \rightarrow \mathcal{X}$ be a covering morphism of formal orbifolds. Then $f$ induces a homomorphism of their fundamental group $\pi_{1}(f): \pi_{1}(\mathcal{Y}) \rightarrow$ $\pi_{1}(\mathcal{X})$. This makes $\pi_{1}$ a functor from connected pointed formal orbifolds to groups. Moreover if $f: \mathcal{Y} \rightarrow \mathcal{X}$ is étale then $\pi_{1}(f)$ is injective.

Proof. The proof is same as that of $[\mathrm{KP}$, Proposition 2.26].

Remark 5.3. Let $P_{1} \geq P_{2}$ be branch data on connected $X$. Then $i d:\left(X, P_{1}\right) \rightarrow$ $\left(X, P_{2}\right)$ is a covering morphism of orbifolds. The induced map on their fundamental groups $\pi_{1}(i d): \pi_{1}\left(X, P_{1}\right) \rightarrow \pi_{1}\left(X, P_{2}\right)$ is an epimorphism.

Theorem 5.4. Let $X$ be a normal connected variety over a perfect field or $\operatorname{Spec}(\mathbb{Z})$ and $U$ an open subset of $X$. Then $\pi_{1}(U) \cong \lim \pi_{1}(X, P)$ where $P$ runs over all the branch data on $X$ such that $\mathrm{BL}(P) \subset X \backslash U$. 
Proof. Let $D=X \backslash U$. Since compositum $P_{0}$ of two branch data $P_{1}, P_{2}$ is a branch data on $X$ with $P_{0} \geq P_{i}, i=1,2$ and $\operatorname{BL}\left(P_{1} P_{2}\right)=\operatorname{BL}\left(P_{1}\right) \cup \operatorname{BL}\left(P_{2}\right)$. The collection of branch data with $\mathrm{BL}(P) \subset D$ is a projective system. Also if $P_{1} \geq P_{2}$ are two branch data on $X$ then the $i d_{X}:\left(X, P_{2}\right) \rightarrow\left(X, P_{1}\right)$ is a covering morphism. By functoriality (Proposition 5.2) it induces a group homomorphism $\pi_{1}\left(X, P_{2}\right) \rightarrow \pi_{1}\left(X, P_{1}\right)$. Hence $\pi_{1}(X, P)$ where $P$ runs over all the branch data on $X$ such that $\operatorname{BL}(P) \subset D$ is an inverse system of groups.

Note that $\pi_{1}(U)=\varliminf_{\mathrm{L}} \operatorname{Aut}(L / k(U))$ where $L$ varies over all finite field extensions $L / k(U)$ (in a fixed separable closure) such that the normalization $V$ of $U$ in $L$ is étale over $U$. Any such extension $L / k(U)$ correspond to a finite morphism $f: Y \rightarrow X$ which extends $V \rightarrow U$. Note that $f:(Y, O) \rightarrow\left(X, B_{f}\right)$ is an étale covering morphism by Corollary 4.12 and $\mathrm{BL}\left(B_{f}\right) \subset D$ as it is the branch locus of $f$ and $f$ is étale over $U$. Conversely given an étale covering morphism $f:(Y, Q) \rightarrow(X, P)$ with $\mathrm{BL}(P) \subset D, f$ restricted to $V:=f^{-1}(U)$ is étale. Hence the two inverse limits are isomorphic.

Proposition 5.5. If $\left(X, P_{1}\right)$ and $\left(X, P_{2}\right)$ are geometric formal orbifolds then so is $\left(X, P_{1} P_{2}\right)$.

Proof. Let $f_{1}:\left(Y_{1}, Q_{1}\right) \rightarrow\left(X, P_{1}\right)$ and $f_{2}:\left(Y_{2}, Q_{2}\right) \rightarrow\left(X, P_{2}\right)$ be étale covering morphisms with $Q_{1}$ and $Q_{2}$ essentially trivial branch data. Let $P=P_{1} P_{2}$ and $R_{i}=f_{i}^{*} P$. Then $f_{i}:\left(Y_{i}, R_{i}\right) \rightarrow(X, P)$ are both étale. Let $(W, A)$ be the fiber product of $\left(Y_{i}, R_{i}\right) \rightarrow(X, P)$ for $i=1,2$. For a point $w$ in $W$ with images $y_{i}$ in $Y_{i}$, $i=1,2$ and $x \in X, A(w)=R_{1}\left(y_{1}\right) R_{2}\left(y_{2}\right) \mathcal{K}_{W, w}$. For $i=1,2$, since $Q_{i}$ is essentially trivial branch data on $Y_{i}, Q_{i}\left(y_{i}\right) / \mathcal{K}_{Y, y_{i}}$ is unramified and hence $Q_{i}\left(y_{i}\right) \mathcal{K}_{W, w} / \mathcal{K}_{W, w}$ is unramified. So the compositum $Q_{1}\left(y_{1}\right) Q_{2}\left(Y_{2}\right) \mathcal{K}_{W, w}$ is an unramified extension of $\mathcal{K}_{W, w}$. But $R_{i}\left(y_{i}\right) \subset Q_{i}\left(y_{i}\right)$. Hence $R_{1}\left(y_{1}\right) R_{2}\left(y_{2}\right) \mathcal{K}_{W, w} / \mathcal{K}_{W, w}$ is unramified. Hence $A$ is essentially trivial branch data and by Corollary $4.18(W, A) \rightarrow(X, P)$ is étale.

Proposition 5.6. Let $(X, P)$ be a connected formal orbifold. There exists $Q \leq P$ a (unique) branch data on $X$ such that $(X, Q)$ is a geometric formal orbifold and $Q$ is maximal with these properties. The natural homomorphism $\pi_{1}(X, P) \rightarrow \pi_{1}(X, Q)$ is an isomorphism.

Proof. Let $f:(Y, R) \rightarrow(X, P)$ be a connected Galois étale covering morphism. Let $B_{f}$ be the branch data on $X$. Then $B_{f} \leq P$. Let $p_{1}, \ldots, p_{r}$ be the irreducible components of $\operatorname{Supp}(P)$. Let $Q\left(p_{j}\right)$ be the compositum of all $B_{f}\left(p_{j}\right)$ where $f$ : $(Y, R) \rightarrow(X, P)$ is Galois étale. Then for all $1 \leq j \leq r, \mathcal{K}_{X, p_{j}} \subset Q\left(p_{j}\right) \subset P\left(p_{j}\right)$ and $Q\left(p_{j}\right) / \mathcal{K}_{X, p_{j}}$ is a finite Galois extension. So there exist finitely many $f_{i}:\left(Y_{i}, R_{i}\right) \rightarrow$ $(X, P)$ étale connected Galois covering such that $Q\left(p_{j}\right)=B_{f_{1}}\left(p_{j}\right) \ldots B_{f_{n}}\left(p_{j}\right)$ for $1 \leq j \leq r$. Let $Q=B_{f_{1}} \ldots B_{f_{n}}$. Then $Q \leq P, Q$ is a geometric branch data by Proposition 5.5 and Corollary 4.12. By construction of $Q$ it is maximal geometric branch data bounded by $P$ and the homomorphism $\pi_{1}(X, P) \rightarrow \pi_{1}(X, Q)$ is an isomorphism.

Corollary 5.7. Let $(X, P)$ be a connected formal orbifold then $\pi_{1}(X, P)$ is an extension of a finite group by $\pi_{1}\left(X^{\prime}\right)$ the étale fundamental group of a connected normal variety $X^{\prime}$.

Proof. By Proposition 5.6 there exists a geometric branch data $Q$ on $X$ such that $\pi_{1}(X, P)=\pi_{1}(X, Q)$. Since $(X, Q)$ is a geometric formal orbifold there exists a 
finite étale Galois covering $f:(Y, R) \rightarrow(X, Q)$ (with Galois group $G$ say) where $R$ is an essentially trivial branch data. Then $\pi_{1}(Y, R)$ is a normal subgroup of $\pi_{1}(X, Q)$ with quotient isomorphic to $G$ (Proposition 5.2). Since $R$ is essentially trivial branch data $\pi_{1}(Y, R)=\pi_{1}(Y)$.

Recall that a profinite group $\Pi$ is said to be of finite rank if there exists a finite subset $S$ of $\Pi$ such that the subgroup generated by $S$ is dense in $\Pi$.

Corollary 5.8. Let $(X, P)$ be a connected formal orbifold with $X$ projective over $\operatorname{Spec}(k)$ where the field $k$ is such that the absolute Galois group $G_{k}$ has finite rank then $\pi_{1}(X, P)$ has finite rank.

Proof. It follows from the above corollary and the fact that $\pi_{1}\left(X^{\prime}\right)$ is topologically finitely generated for any connected normal projective variety $X^{\prime}$ over $\operatorname{Spec}(k)$. (This is true for curves via Grothendieck lifting. For higher dimensional normal projective variety $X^{\prime}$ one uses Lefschetz's theorem for fundamental groups, i.e. there exists a general projective curve $C$ in $X^{\prime}$ such that $\pi_{1}(C) \rightarrow \pi_{1}\left(X^{\prime}\right)$ is surjective.)

\section{6. Étale Sheaves AND COHOMOLOGy}

In this section using the notion of étale morphism for formal orbifolds we briefly define small étale site on formal orbifolds, sheaves on them and their cohomology groups using the standard technique (see [Mil, Chapter II and III] or [SP, Chapter 20 and 21]). We also prove some of the results in the formal orbifold setup which will be used in the later sections. Note that even if $f:(Y, Q) \rightarrow(X, P)$ is an étale morphism of formal orbifolds, $f: Y \rightarrow X$ viewed as a morphism of varieties need not be flat.

Let $\mathcal{X}=(X, P)$ be a formal orbifold. An étale $\mathcal{X}$-formal orbifold is an étale morphism $f:(Y, Q) \rightarrow \mathcal{X}$ and morphisms between étale $\mathcal{X}$-formal orbifolds is an étale $X$-morphism. This category will be denoted by $E / \mathcal{X}$. An open étale cover of $\mathcal{X}$ is a collection of étale morphisms $\left\{f_{i}:\left(U_{i}, Q_{i}\right) \rightarrow \mathcal{X}, i \in I\right\}$ such that $\cup_{i \in I} f_{i}\left(U_{i}\right)=X$. This defines an étale topology on the category of étale $\mathcal{X}$-formal orbifolds. In other words this defines a small étale site $E / \mathcal{X}$ on $\mathcal{X}$.

As usual a presheaf on $E / \mathcal{X}$ is defined to be a contravariant functor from $E / \mathcal{X}$ to the category of abelian groups. A morphism $\phi$ of presheaves $\mathcal{F}$ and $\mathcal{G}$ is a natural transformation of functors where maps $\phi_{U}: \mathcal{F}(U) \rightarrow \mathcal{G}(U)$ for an object $U$ in $E / \mathcal{X}$ is a group homomorphism. Note that the category of presheaves is an abelian category. The argument is same as in the usual Grothendieck topology on schemes and just uses the fact that this category of functors shares most of the properties of the category of abelian groups.

A sheaf $\mathcal{F}$ is a presheaf such that for any $\mathcal{U}$ in $E / X$ and any étale cover $\left\{f_{i}\right.$ : $\left.\mathcal{U}_{i} \rightarrow \mathcal{U}\right\}$, the following sequence is exact.

$$
F(\mathcal{U}) \rightarrow \Pi_{i} F\left(\mathcal{U}_{i}\right) \rightrightarrows \Pi_{i, j} F\left(\mathcal{U}_{i} \times \mathcal{U} \mathcal{U}_{j}\right)
$$

Example. Let $A$ be an abelian group. Consider the presheaf $\mathcal{F}_{A}$ on $\mathcal{X}$ which sends $\mathcal{U} \rightarrow \mathcal{X}$ to $\mathcal{F}(\mathcal{U})=\{s: \mathcal{U} \rightarrow A \mid s$ is continuous $\}$ where we give $A$ the discrete topology and $\mathcal{U}=(U, P)$ the Zariski topology on $U$. And which sends an étale $\mathcal{X}$ morphism between étale $\mathcal{X}$-formal orbifolds $f: \mathcal{U} \rightarrow \mathcal{V}$ to the group homomorphism $\mathcal{F}_{A}(f): \mathcal{F}_{A}(\mathcal{V}) \rightarrow \mathcal{F}_{A}(\mathcal{U})$ where $\mathcal{F}_{A}(f)(s)=s \circ f$. The gluing condition (6.1) for such a presheaf is automatic hence it is a sheaf and it will be denoted by $A$ as well 
and will be called a constant sheaf. Note that $\mathcal{F}_{A}(\mathcal{U})$ is isomorphic to $r$ copies of $A$ where $r$ is the number of connected components of $\mathcal{U}$.

6.1. Čech cohomology. Let $\mathcal{F}$ be a presheaf on $\mathcal{X}$ and $\mathscr{U}=\left\{\mathcal{U}_{i} \rightarrow \mathcal{X}\right\}$ be a covering. We define the Cech cohomology groups $\check{H}^{*}(\mathscr{U}, \mathcal{F})$ of $\mathcal{F}$ with respect to the covering $\mathscr{U}$ in the usual way. For $n \geq 0$, let $\mathcal{U}_{i_{0}, \ldots, i_{n}}=\mathcal{U}_{i_{0}} \times \mathcal{X} \mathcal{U}_{i_{1}} \times \mathcal{X} \ldots \times \mathcal{X}_{i_{n}}$ denote the $n$-fold intersection. Consider the Čech complex $C^{*}(\mathscr{U}, \mathcal{F})$ whose $n^{\text {th }}$ term is $C^{n}=\Pi_{i_{0}<\ldots<i_{n}} \mathcal{F}\left(\mathcal{U}_{i_{0}, \ldots, i_{n}}\right)$.

The boundary maps $d: C^{n} \rightarrow C^{n+1}$ are given by the alternating sum of the natural maps $\mathcal{F}\left(\mathcal{U}_{i_{0}, \ldots, \hat{i}_{k}, \ldots, i_{n}}\right) \rightarrow \mathcal{F}\left(\mathcal{U}_{i_{0}, \ldots, i_{n}}\right)$ induced from the natural projection morphism $\mathcal{U}_{i_{0}, \ldots, i_{n}} \stackrel{\mathcal{L}}{\rightarrow} \mathcal{U}_{i_{0}, \ldots, \hat{i}_{k}, \ldots, i_{n}}$.

The homology of this complex is $\check{H}^{*}(\mathscr{U}, \mathcal{F})$. If $\mathscr{V}=\left\{\mathcal{V}_{i} \rightarrow \mathcal{X}\right\}$ is another covering of $\mathcal{X}$ and a refinement of $\mathscr{U}$. Then there is a map of complexes $C^{*}(\mathscr{U}, \mathcal{F}) \rightarrow$ $C^{*}(\mathscr{V}, \mathcal{F})$ which induces maps on the cohomology groups $\check{H}^{*}(\mathscr{U}, \mathcal{F}) \rightarrow \check{H}^{*}(\mathscr{V}, \mathcal{F})$. This forms a directed system of groups as the coverings of $\mathcal{X}$ vary. Define Cech cohomology groups of $X$ for a presheaf $\mathcal{F}$ as:

$$
\check{H}^{*}(X, \mathcal{F})=\underset{\mathscr{U}}{\lim } H^{*}(\mathscr{U}, \mathcal{F}) .
$$

Note that for a sheaf $\mathcal{F}, H^{0}(\mathcal{X}, \mathcal{F})=\mathcal{F}(\mathcal{X})$. For a presheaf $\mathcal{F}$, define $\mathcal{F}^{+}$to be the functor $\mathcal{F}^{+}(\mathcal{U})=\check{H}^{0}\left(\mathcal{U},\left.\mathcal{F}\right|_{\mathcal{U}}\right)$. There is a natural map of presheaves $\mathcal{F} \rightarrow \mathcal{F}^{+}$.

Theorem 6.1. [SP, Theorem 7.10.10] Let $\mathcal{F}$ be a presheaf on $\mathcal{X}$. Then

(1) The presheaf $\mathcal{F}^{+}$is a separated presheaf.

(2) If $\mathcal{F}$ is a separated presheaf then $\mathcal{F}^{+}$is a sheaf and the natural map $\mathcal{F} \rightarrow \mathcal{F}^{+}$ is injective.

(3) If $\mathcal{F}$ is a sheaf then the natural map $\mathcal{F} \rightarrow \mathcal{F}^{+}$is an isomorphism.

(4) The presheaf $\mathcal{F}^{++}$is always a sheaf and $\mathcal{F} \rightarrow \mathcal{F}^{++}$is called the sheafification of $\mathcal{F}$.

Let $A$ be an abelian group. The constant presheaf described in Example 6 is a sheaf. So $\check{H}^{0}(\mathcal{X}, A)=A^{r}$ where $r$ is the number of connected component of $\mathcal{X}$.

Proposition 6.2. Let $\mathcal{X}=(X, P)$ be a formal orbifold such that $X$ is connected and projective over a field $k$ and $G_{k}$ is a finite rank profinite group. Then $\check{H}^{1}(\mathcal{X}, A) \cong$ $\operatorname{Hom}\left(\pi_{1}(\mathcal{X}), A\right)$ for any finite abelian group $A$.

Proof. Since $A$ is a finite abelian group and $\pi_{1}(\mathcal{X})$ is finite rank (by Corollary 5.8) $\operatorname{Hom}\left(\pi_{1}(\mathcal{X}), A\right)$ is a finite group. Hence there exists a finite $G$-Galois étale cover $\mathcal{Y} \rightarrow \mathcal{X}$ such that every element of $\operatorname{Hom}\left(\pi_{1}(\mathcal{X}), A\right)$ factors through $G$. As in [Mil, Chapter III, Example 2.6, page 99] the Čech complex with respect to the cover $\{\mathcal{Y} \rightarrow \mathcal{X}\}$ of $A$ is isomorphic to cochain complex used to compute the group cohomology $H^{*}(G, A)$. Hence $H^{1}(\mathcal{Y} \rightarrow \mathcal{X}, A) \cong H^{1}(G, A)$. Since $A$ is a constant sheaf $G$ acts trivially on $A$ and hence $H^{1}(G, A)=\operatorname{Hom}(G, A)$. But $\operatorname{Hom}(G, A)$ is same as $\operatorname{Hom}\left(\pi_{1}(\mathcal{X}), A\right)$ by choice of $Y$ and $G$. Note that any refinement of $\mathcal{Y} \rightarrow \mathcal{X}$ has a further refinement by a $G^{\prime}$-Galois cover $\mathcal{Y}^{\prime} \rightarrow \mathcal{X}$ and hence dominates $\mathcal{Y} \rightarrow \mathcal{X}$. So by the same argument $\check{H}^{1}\left(\mathcal{Y}^{\prime} \rightarrow \mathcal{X}, A\right) \cong \operatorname{Hom}\left(\pi_{1}(\mathcal{X}), A\right)$. Hence $\check{H}^{1}(\mathcal{X}, A) \cong \operatorname{Hom}\left(\pi_{1}(\mathcal{X}), A\right)$.

Remark 6.3. This result holds without the assumption on the base field and the assumption on $X$ is projective. Note that in absence of these assumptions $\operatorname{Hom}\left(\pi_{1}(\mathcal{X}), A\right)$ will not be a finite group. But $\pi_{1}(\mathcal{X})$ is a Galois group of an 
infinite Galois extension. Hence one has to work with a inverse system of finite Galois covers and modify the argument in [Mil, Chapter III, Example 2.6, page 99] to get the result in this general setup.

\subsection{Structure sheaf.}

Lemma 6.4. Let $A \rightarrow B$ be a finite separable extension of normal domains. Then the sequence $A \rightarrow B \rightrightarrows B \times{ }_{A} B$ is exact.

Proof. Since $A \rightarrow B$ is generically étale there exists a multiplicative subset $S$ in $A$ such that $S^{-1} A \rightarrow S^{-1} B$ is a finite étale morphism. Hence the sequence $S^{-1} A \rightarrow$ $S^{-1} B \rightrightarrows S^{-1} B \otimes_{S^{-1} A} S^{-1} B$ is exact. Note that $A$ is contained in the kernel of the map $B \rightarrow B \otimes_{A} B$ which sends $b \in B$ to $1 \otimes b-b \otimes 1$. So it is enough to show that if $b \in B$ is such that $b \otimes 1-1 \otimes b=0$ in $B \otimes_{A} B$ then $b \in A$. But for such a $b$, the exactness after localization implies that $b \in S^{-1} A$. But $b$ is integral over $A$ and $A$ is normal implies that $b \in A$.

Let $\mathcal{X}=(X, P)$ be a formal orbifold. Consider the structure presheaf $\mathcal{O}_{\mathcal{X}}$ which sends $\mathcal{U}=(U, Q)$ to $\mathcal{O}_{U}(U)$.

Proposition 6.5. The structure presheaf $\mathcal{O}_{\mathcal{X}}$ of a formal orbifold is a sheaf.

Proof. Let $\left(Y_{i}, Q_{i}\right) \rightarrow(X, P)$ be a covering then $\amalg Y_{i} \rightarrow X$ is a finite separable morphism of normal schemes. Hence the exactness of the sheaf condition for the structure presheaf follows from the above lemma and Mil, Chapter II, Proposition $1.5]$.

Once the structure sheaf of a formal orbifold $\mathcal{X}$ is defined, sheaf of $\mathcal{O}_{\mathcal{X}}$-modules are simply sheaf of abelian groups $\mathcal{F}$ such that $\mathcal{F}(\mathcal{U})$ is an $\mathcal{O}_{\mathcal{X}}(\mathcal{U})$-module for every étale morphism $\mathcal{U} \rightarrow \mathcal{X}$.

6.3. Pullback and pushforward. The pullback and the pushforward of sheaves on formal orbifolds with respect to admissible morphisms of formal orbifolds can be defined in the same way as for the schemes in [Mil, Chapter II].

Let $f: \mathcal{Y} \rightarrow \mathcal{X}$ be an admissible morphism of formal orbifolds. Let $\mathcal{F}$ be a presheaf on $\mathcal{Y}$ then $f_{*} \mathcal{F}$ is the presheaf given by the functor which sends $\mathcal{U}$ in $E / \mathcal{X}$ to $\mathcal{F}(\mathcal{U} \times \mathcal{X} \mathcal{Y})$. This functor from the category of presheaves on $\mathcal{X}$ to the category of presheaves on $\mathcal{Y}$ is called a direct image functor. Its adjoint $f^{p}$ is a functor from presheaves on $\mathcal{X}$ to presheaves on $\mathcal{Y}$ called the inverse image functor. If $\mathcal{F}$ is a sheaf then $f_{*} \mathcal{F}$ is also a sheaf (cf. [Mil, Chapter II, Proposition 2.7]). The sheaf $f_{*} \mathcal{F}$ is also called the pushforward of $\mathcal{F}$. But $f^{p}$ need not send sheaves to sheaves and hence the pullback is defined as the sheafification of the inverse image functor.

6.4. Sheaf cohomology. The étale site $E / \mathcal{X}$ on a formal orbifold $\mathcal{X}$ has a final object and hence the global section functor $\Gamma(\mathcal{X},-)$ from the category of sheaves of abelian groups on $\mathcal{X}, \operatorname{Sh}(\mathcal{X})$ to the category of abelian groups $A b$ is given by $\mathcal{F} \mapsto \mathcal{F}(\mathcal{X})$. By [SP, Theorem 19.7.4] there are enough injectives in $\operatorname{Sh}(\mathcal{X})$. Hence we can define the sheaf cohomology $H^{i}(\mathcal{X}, \mathcal{F})$ as the right derived functor of the global section functor. As usual the Cech cohomology and the sheaf cohomology agree for degree 0 and 1 ([Mil, Chapter III, Corollary 2.10]). Hence in view of Theorem 6.2 we get that $H^{1}(\mathcal{X}, A) \cong \operatorname{Hom}\left(\pi_{1}(\mathcal{X}), A\right)$.

One could directly show the isomorphism $H^{1}(\mathcal{X}, A) \cong \operatorname{Hom}\left(\pi_{1}(\mathcal{X}), A\right)$. This can be done using Hochschild-Serre spectral sequence imitating the proof in the étale case as carried out in Mil, Chapter III, Theorem 2.20 and Remark 2.21(b)]. 
Proposition 6.6. Let $\mathcal{X}=(X, P)$ be a geometric formal orbifold and $f:(Y, O) \rightarrow$ $(X, P)$ be a finite étale Galois $G$-cover. Let $\mathcal{F}$ be a sheaf on $\mathcal{X}$. Then there is a spectral sequence

$$
H^{p}\left(G, H^{q}\left(Y,\left.\mathcal{F}\right|_{Y}\right)\right) \Rightarrow H^{p+q}(\mathcal{X}, \mathcal{F})
$$

Here $H^{q}\left(Y,\left.\mathcal{F}\right|_{Y}\right)$ is the usual étale cohomology.

Proof. The global section functor $\Gamma(\mathcal{X},-)$ from $\operatorname{Sh}(\mathcal{X})$ to abelian groups is the composition $(-)^{G} \circ \Gamma(Y,-)$ of global sections over $Y$ followed by $G$-invariants. Note that $G$ acts naturally of $\left.\mathcal{F}\right|_{Y}$ and hence $\Gamma(Y,-)$ is a functor from $\operatorname{Sh}(\mathcal{X})$ to $G$-modules. Note that $\Gamma(Y,-)$ takes injective objects in $\operatorname{Sh}(\mathcal{X})$ to injective objects in the category of $G$-modules. The result now follows from Grothendieck spectral sequence.

Remark 6.7. Since $Y$ has finite cohomological dimension. This is a bounded spectral sequence.

Corollary 6.8. Let $\mathcal{X}$ be a proper geometric formal orbifold and $\mathcal{F}$ on $X$ be such that $\left.\mathcal{F}\right|_{Y}$ is coherent for some étale Galois cover $(Y, O) \rightarrow \mathcal{X}$. Then $H^{i}(\mathcal{X}, \mathcal{F})$ is finite dimensional for all $i$.

Proof. Note that if $X$ is proper over $k$ then $Y$ is also proper over $k$. In this situation, $H^{q}\left(Y,\left.\mathcal{F}\right|_{Y}\right)$ are finite dimensional if $\left.\mathcal{F}\right|_{Y}$ is coherent and hence $H^{i}(\mathcal{X}, \mathcal{F})$ are also finite dimensional.

\section{BRYLINSKi-KATO FILTRATION}

We recall the Brylinski-Kato filtration on the ring of finite length Witt vectors over a henselian discrete valuation field $K$ of characteristic $p$ from [Kat, Mat and KS2]. For $m \geq 0$,

$$
f i l_{m}^{\log } W_{s}(K)=\left\{\left(a_{s-1}, \ldots, a_{0}\right) \in W_{s}(K) \mid p^{i} \underset{K}{\operatorname{ord}}\left(a_{i}\right) \geq-n \forall i\right\}
$$

Note that this is an increasing filtration with $f i l_{0}^{l o g} W_{s}(K)=W_{s}\left(\mathcal{O}_{K}\right)$. A modification of this filtration by Matsuda was used in [KS2]. Let $V: W_{s}(K) \rightarrow W_{s+1}(K)$ be the function sends $\left(a_{s-1}, \ldots, a_{0}\right)$ to $\left(0, a_{s-1}, \ldots, a_{0}\right)$ called the Verschiebung. Let $s^{\prime}=\min \left(\operatorname{ord}_{p}(m+1), s\right)$ then define

$$
f i l_{m} W_{s}(K)=V^{s-s^{\prime}} f i l_{m+1}^{\log } W_{s^{\prime}}(K)+f i l_{m}^{\log } W_{s}(K) .
$$

Let $\delta_{s}: W_{s}(K) /(F r o b-I d) \rightarrow H^{1}\left(K, \mathbb{Z} / p^{s} \mathbb{Z}\right)$ be the isomorphism given by ArtinSchreier-Witt correspondence. The filtration on the Witt rings induce a filtration on $H^{1}(K):=H^{1}(K, \mathbb{Q} / \mathbb{Z})$ as follows:

$$
f i l_{m} H^{1}(K)=H^{1}(K)\left(p^{\prime}\right)+\cup_{s \geq 1} \delta_{s}\left(f i l_{m} W_{s}(K)\right) \text { for } m \geq 1 .
$$

Let $f i l_{0} H^{1}(K)$ be the subgroup of unramified characters. This filtration has a shift in numbering from [Mat but it is consistent with [KS2] and [KS1].

Definition 7.1. (1) For a finite abelian field extension $L / K$ of $p$-exponent less than $n$, let $W_{L}$ be the subgroup of $W_{n}(K) / F r o b-I d$ corresponding to the $p$-part of the extension $L / K$ via the Artin-Schreier-Witt theory. Define the swan conductor

$$
S w(L / K)=\min \left\{m: W_{L} \subset f i l_{m} W_{n}(K) /(F r o b-I d)\right\} .
$$


(2) For a branch data $P$ on a normal variety $X$, define the swan divisor of $(X, P)$

$$
S w(X, P)=\sum_{x \in X^{(1)}} S w\left(P(x) / \mathcal{K}_{X, x}\right) x,
$$

where $X^{(1)}$ is the set of codimension one points in $X$.

Let $\mathcal{X}=(X, P)$ be a formal orbifold over a perfect field $k$. Assume that $P(x) / \mathcal{K}_{X, x}$ is an abelian extension for all $x \in X$. Let $U=X \backslash \operatorname{BL}(P)$. Set $H^{1}(\mathcal{X}):=\lim _{n} H^{1}(\mathcal{X}, \mathbb{Z} / n \mathbb{Z})$. Note that $\pi_{1}^{a b}(\mathcal{X}) \cong \operatorname{Hom}\left(H^{1}(\mathcal{X}), \mathbb{Q} / \mathbb{Z}\right)$ by Pontriagan duality. Let $D$ be an effective Cartier divisor on $X$ supported on $\operatorname{BL}(P)$. We recall the definition of $f i l_{D} H^{1}(U)$ as in [KS1.

Definition 7.2. For $\chi \in H^{1}(U)$ to be in $f i l_{D} H^{1}(U)$ the following must be true. For all integral curves $Z$ in $X$ not contained in $\operatorname{BL}(P)$ and for any closed point $x$ in the normalization $\bar{Z}$ of $Z$ which lies above $Z \cap \mathrm{BL}(P), \chi \mid G_{x} \in f i l_{m(x, D)} H^{1}\left(\mathcal{K}_{\bar{Z}, x}\right)$. Here $G_{x}=\operatorname{Gal}\left(\mathcal{K}_{\bar{Z}, x}^{s} / \mathcal{K}_{\bar{Z}, x}\right)$ and $m(x, D)$ is the multiplicity of $x$ in the pullback of $D$ under the composition $\bar{Z} \rightarrow Z \rightarrow X$.

Definition 7.3. Define $\pi_{1}^{a b}(X, D)=\operatorname{Hom}\left(f i l_{D} H^{1}(U), \mathbb{Q} / \mathbb{Z}\right)$. This is a quotient of $\pi_{1}^{a b}(U)$ and corresponds to abelian covers of $X$ étale over $U$ whose ramification over $\mathrm{BL}(P)$ is "bounded by $D$ ".

Theorem 7.4. Let $\mathcal{X}=(X, P)$ be a proper connected formal orbifold such that $Z=\mathrm{BL}(P)$ is a simple normal crossing divisor on $X$ and let $U=X \backslash Z$. The image of the inclusion $H^{1}(\mathcal{X}) \rightarrow H^{1}(U)$ lies in fil $_{D} H^{1}(U)$ where the divisor $D$ on $X$ is given by $D=S w(X, P)$. Moreover for a Cartier divisor $D$ on $X$ whose support is a normal crossing divisor,

$$
\pi_{1}^{a b}(X, D)=\lim _{\longleftarrow} \pi_{1}^{a b}(X, Q)
$$

where $Q$ varies over all branch data such that $Q$ is abelian and $S w(X, Q) \leq D$.

Proof. Let $\chi$ be in the image of $H^{1}(\mathcal{X}) \rightarrow H^{1}(U)$. Let $K=k(U), K^{u r, \mathcal{X}, a b}$ be the compositum of function fields of abelian étale covers of $\mathcal{X}$ and $K^{u r, U, a b}$ be the compositum of function fields of abelian étale covers of $U$. Then $\pi_{1}^{a b}(\mathcal{X})=$ $\operatorname{Gal}\left(K^{u r, \mathcal{X}, a b} / K\right)$ and $\pi_{1}^{a b}(U)=\operatorname{Gal}\left(K^{u r, U, a b} / K\right)$.

The hypothesis on the character $\chi \in \operatorname{Hom}\left(\pi_{1}^{a b}(U), \mathbb{Q} / \mathbb{Z}\right)$ implies that it factors through $\pi_{1}^{a b}(\mathcal{X})$. Let $x \in X$ be a codimension one point lying in $\operatorname{BL}(P)$. Note that the abelian part of the decomposition group at $x, G_{x}=\operatorname{Gal}\left(\mathcal{K}_{X, x} K^{u r, U, a b} / \mathcal{K}_{X, x}\right) \leq$ $\pi_{1}^{a b}(U)$. Note that the natural map $H^{1}(U) \rightarrow H^{1}\left(\mathcal{K}_{X, x}\right)$ is given by $\chi \mapsto \chi_{x}:=$ $\left.\chi\right|_{G_{x}}$.

In view of [KS1, Proposition 2.5], to show that $\chi \in f i l_{D} H^{1}(U)$, it is enough to show $\chi_{x} \in f i l_{m_{x}} H^{1}\left(\mathcal{K}_{X, x}\right)$ where $m_{x}=S w\left(P(x) / \mathcal{K}_{X, x}\right)$. Again since $\chi$ factors through $\pi_{1}^{a b}(\mathcal{X}), \chi_{x}$ factors through $\operatorname{Gal}\left(\mathcal{K}_{X, x} K^{u r, \mathcal{X}, a b} / \mathcal{K}_{X, x}\right)$. But $\mathcal{K}_{X, x} K^{u r, \mathcal{X}, a b}=$ $P(x) L$ where $L / \mathcal{K}_{X, x}$ is an unramified extension (over $\widehat{\mathcal{O}}_{X, x}$ ). Hence

$$
\chi_{x} \in \cup_{s} \delta_{s}\left(W_{P(x)}\right)+f i l_{0} H^{1}\left(\mathcal{K}_{X, x}\right)
$$

because by definition of Brylinski-Kato filtration on $H^{1}\left(\mathcal{K}_{X, x}\right)$ the unramified characters make up $f i l_{0} H^{1}\left(\mathcal{K}_{X, x}\right)$. Hence $\chi_{x} \in f i l_{m} H^{1}(U)$ if $W_{P(x)} \subset f i l_{m} W_{s}\left(\mathcal{K}_{X, x}\right)$ for some $s$. And this happens if $m \geq m_{x}=S w\left(P(x) / \mathcal{K}_{X, x}\right)$.

For the moreover part, let $U=X \backslash \operatorname{Supp}(D)$. Note that given any $n \geq 1$ and any $\alpha \in f i l_{D} H^{1}(U, \mathbb{Z} / n \mathbb{Z})$ there exists a branch data $Q$ on $X$ with $S w(X, Q) \leq D$ 
such that $\alpha$ is the image of $\left.H^{1}((X, Q), \mathbb{Z} / n \mathbb{Z}) \rightarrow H^{1}(U, \mathbb{Z} / n \mathbb{Z})\right)$. The result now follows by taking the limit and applying Pontriagan duality.

It leads to some natural questions for formal orbifolds.

Question 7.5. Let $\mathcal{X}$ be a geometrically connected proper geometric formal orbifold over a finite field. Does class field theory hold for for $\mathcal{X}$ ?

A geometric definition of chow groups (of zero cycles) for formal orbifolds is needed to make this more precise. One way to approach this problem is to realize that $\pi_{1}^{a b}(\mathcal{X})$ is a quotient of $\pi_{1}^{a b}(X, D)$ for $D=S w(\mathcal{X})$. So by Kerz-Saito's class field theory chow groups of zero cycles on $\mathcal{X}$ should be an appropriate quotient of chow groups of $X$ with modulus as defined in [KS2]. And one could try to provide a geometric or hopefully a motivic interpretation of the this quotient group directly in terms of $\mathcal{X}$.

It will be desirable to obtain a class field theory for formal orbifolds such that the inverse limits of the cycle class maps yield the cycle class map of Kerz and Saito's class field theory. A starting point would be to show a version Lefschetz's theorem on fundamental groups for formal orbifolds. For $\pi_{1}^{a b}(X, D)$ it was proved by Kerz and Saito in KS1.

\section{GrothendiecK-LefschetZ theOREM For Fundamental GROUPS}

For a formal orbifold $(X, P)$, let $\operatorname{Cov}(X, P)$ denote the category of finite étale covers $(X, P)$. The question of interest in this section is the following:

Question 8.1. Let $\mathcal{X}=(X, P)$ be a geometrically connected projective smooth geometric formal orbifold of dimension $n \geq 2$ over a perfect field $k$. Does there exist a connected smooth hypersurface $Y$ on $X$ with a geometric branch data $Q$ such that the functor from $\operatorname{Cov}(X, P) \rightarrow \operatorname{Cov}(Y, Q)$, given by the normalized pullback, is fully faithful? In particular, is the induced homomorphism $\pi_{1}(Y, Q) \rightarrow \pi_{1}(X, P)$ surjective? Moreover, if dimension $n>2$ then can one find a $(Y, Q)$ such that the functor $\operatorname{Cov}(X, P) \rightarrow \operatorname{Cov}(Y, Q)$ is an equivalence? In particular, is the map $\pi_{1}(Y, Q) \rightarrow \pi_{1}(X, P)$ an isomorphism?

The above question can be considered as the Grothendieck-Lefschetz theorem for wildly ramified covers. In this section we make some progress towards answering this question.

Let $Y$ be a normal connected hypersurface in $X$ not contained in BL $(P)$. Let $\mathfrak{p}$ be the ideal sheaf on $X$ defining $Y$. Since $Y$ is normal and hence unibranched for any codimension one point $x \in Y$ and any affine connected neighbourhood $U$ of $x$

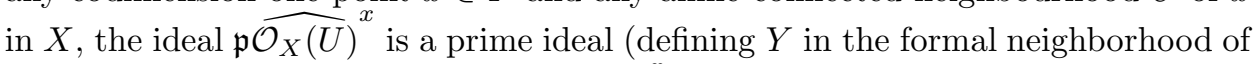
$x$ ). Let $R$ be the integral closure of $\widehat{\mathcal{O}_{X}(U)}{ }^{x}$ in $P(x, U)$ and $\mathfrak{q}_{1}, \ldots, \mathfrak{q}_{r}$ be the prime ideals of $R$ lying above $\mathfrak{p}$. Define $Q^{\prime}(x, U \cap Y)$ for $x \in Y$ of codimension at least one to be the compositum of the Galois extensions $\mathrm{QF}\left(R / \mathfrak{q}_{i}\right)$ of $\mathrm{QF}\left({\widehat{\mathcal{O}_{X}(U}}^{x} / \mathfrak{p}\right)$. Then it is easy to see that $Q^{\prime}$ is a branch data on $Y$.

Definition 8.2. The branch data $Q^{\prime}$ on $Y$ is called the restriction of the branch data $P$ and is denoted by $P_{\mid Y}$.

Proposition 8.3. Let $(X, P)$ be a formal orbifold and $Y$ be a normal connected hypersurface in $X$ not contained in $\mathrm{BL}(P)$. Let $Q$ be the maximal geometric branch 
data on $Y$ which is less than or equal to $P_{\mid Y}$ (obtained using Proposition [5.6). Let $h:(Z, O) \rightarrow(X, P)$ be an étale covering. Let $W=Y \times_{X} Z, \tilde{W}$ the normalization of $W$ and $g: \tilde{W} \rightarrow Y$ the natural projection morphism then the induced morphism $g:\left(\tilde{W}, g^{*} Q\right) \rightarrow(Y, Q)$ is étale.

Proof. In view of Proposition 4.10, it is enough to show that for any point $w \in \tilde{W}$ of codimension at least one $\mathcal{K}_{\tilde{W}, w} Q(g(w)) / Q(g(w))$ is an unramified extension. Let $x$ and $z$ be the images of $w$ in $X$ and $Z$ respectively under the natural map. Note that $x \in Y$ and $g(w)=h(z)=x$. Note that $\mathcal{O}_{\tilde{W}, w}$ is the normalization of $\mathcal{O}_{Y, x} \otimes_{\mathcal{O}_{X, x}}$ $\mathcal{O}_{Z, z}$. Since $\mathfrak{p}$ is the defining ideal of $Y$ in $X, \mathcal{O}_{Y, x} \otimes_{\mathcal{O}_{X, x}} \mathcal{O}_{Z, z} \cong \mathcal{O}_{Z, z} / \mathfrak{p} \mathcal{O}_{Z, z}$. Hence $\mathcal{O}_{\tilde{W}, w}$ is the normalization $\mathcal{O}_{Z, z} / \mathfrak{p} \mathcal{O}_{Z, z}$. Since $Y \nsubseteq \mathrm{BL}(P)$ and $\mathfrak{q}_{1}, \ldots, \mathfrak{q}_{r}$ are the primes in $\mathcal{O}_{Z, z}$ lying above $\mathfrak{p}, \widehat{\mathcal{O}}_{\tilde{W}, w}$ is the normalization of $\widehat{\mathcal{O}}_{Z, z} / \mathfrak{q}_{i}$ for some $i$. Hence $\mathcal{K}_{\tilde{W}, w}=\mathrm{QF}\left(\widehat{\mathcal{O}}_{Z, z} / \mathfrak{q}_{i}\right) \subseteq P_{\mid Y}(x)$. Since $Q$ is the maximal geometric branch data on $Y$ among those less than or equal to $P_{\mid Y}, \mathcal{K}_{\tilde{W}, w} \subseteq Q(x)$.

Let $\widehat{X}$ be a normal excellent formal scheme. A branch data $\hat{P}$ on $\widehat{X}$ is defined in the same way as for schemes as follows. Let $x$ be a codimension one point of $\widehat{X}$ (i.e. codimension one in the closed fiber) and $\widehat{U}$ be an open affine neighbourhood of $x$ in $\widehat{X}$ given by $\operatorname{Spf}(A)$ then set $\hat{P}(x, \widehat{U})$ is a finite Galois extension of $\operatorname{QF}\left(\widehat{A}^{x}\right)$ where $\widehat{A}^{x}$ is the completion of $A$ w.r.t the prime ideal of $A$ defining the point $x$. The assignment $\hat{P}$ is called a quasi branch data on $\widehat{X}$ if $\hat{P}$ is compatible with affine open neighbourhoods of $x, \widehat{V} \subset \widehat{U} \subset \hat{X}$ and with respect to specialization $x_{1} \subset \overline{\left\{x_{2}\right\}}$ of codimension at least one points of $\widehat{X}$ in the same way as in Definition 3.3 The definition of branch locus $\mathrm{BL}(\hat{P})$ is also the same as in Definition 3.3 and similarly a quasi branch data $\hat{P}$ on $\widehat{X}$ is called a branch data if $\operatorname{BL}(\hat{P})$ is closed in the $\widehat{X}$.

Note that for a point $x \in \operatorname{Spf}(A), I \subset A$ be the ideal of definition of $\operatorname{Spf}(A)$ and $I(x)$ be the ideal of definition of $x$ then $I(x) \supset I$. Let $A$ be a normal excellent ring and $J \supset I$ be ideals of $A$ then ${\widehat{\widehat{A}^{I}}}^{J} \cong \widehat{A}^{J}$. Let $(X, P)$ be a formal orbifold and $Y \subset X$ be a closed irreducible subset not contained in $\operatorname{BL}(P)$. Let $\widehat{X}_{Y}$ be the completion of $X$ along $Y$. The branch data $P$ induces a branch data $\widehat{P}$ on the formal scheme $\widehat{X}_{Y}$. More precisely for a point $y \in Y$ of codimension at least one and $U \subset X$ an affine connected neighbourhood of $y$, define $\widehat{P}(y, U \cap Y)=P(y, U)$. Note that $\left.\widehat{\mathcal{O}_{X}(U)}\right)^{y}=\mathcal{O}_{\widehat{X}_{Y}} \widehat{(U \cap Y)^{y}}$, so $\widehat{P}(y, U)$ is a finite Galois extension of $\mathcal{K}_{\widehat{X}_{Y}}(U \cap Y)^{y}=\mathrm{QF}\left(\widehat{\mathcal{O} X}\left(U^{y}\right)\right.$. Note that $\mathrm{BL}(\widehat{P})=\mathrm{BL}(P) \cap Y$.

Definition 8.4. We will call the pair $\left(\widehat{X}_{Y}, \hat{P}\right)$ as $Y$-adic completion of $(X, P)$. Let $\widehat{X}$ be an excellent normal formal scheme and $\hat{P}$ be a branch data of $\hat{X}$. Like in GM, 3.1.6] a finite morphism of formal scheme is an adic morphism (i.e. pull back of a sheaf of ideal of definition is a sheaf of ideal of definition) of formal schemes which induces a finite morphism on the closed fibers. As mentioned in loc. cit. the category of finite coverings of $\widehat{X}$ is equivalent to the category of sheaf of finite $\mathcal{O}_{\widehat{X}^{-}}$ algebras $\mathcal{A}$ on $\widehat{X}$. Let $f: \widehat{Y} \rightarrow \widehat{X}$ be a finite morphism of excellent normal formal schemes and let $\hat{P}$ and $\hat{Q}$ be branch data on $\widehat{X}$ and $\widehat{Y}$ respectively. Like in Definition 4.1. $f:(\widehat{Y}, \hat{Q}) \rightarrow(\widehat{X}, \hat{P})$ is said to be a cover if for all $y \in \widehat{Y}$ of codimension at least one and some open affine neighbourhood $U$ of $f(y), \hat{Q}\left(y, f^{-1}(U)\right) \supset \hat{P}(f(y), U)$. Moreover the above cover is said to be an étale cover if $\hat{Q}(y)=\hat{P}(f(y))$ for all 
$y \in \widehat{Y}$ of codimension at least one. The category of étale covers of the pair $(\widehat{X}, \hat{P})$ will be denoted by $\operatorname{Cov}(\widehat{X}, \hat{P})$.

8.1. The functor $\operatorname{Cov}\left(\widehat{X}_{Y}, \hat{P}\right)$ to $\operatorname{Cov}\left(Y, P_{\mid Y}\right)$. The pullback along the natural morphism $Y \rightarrow \widehat{X}_{Y}$ defines a functor from $\operatorname{Cov}\left(\widehat{X}_{Y}, \hat{P}\right) \rightarrow \operatorname{Cov}\left(Y, P_{\mid Y}\right)$ (follows in the same way as Proposition 8.3). We shall show that this functor is an equivalence of category when the following hypothesis holds:

Hypothesis 8.5. The formal orbifold $(X, P)$ is geometric, irreducible, smooth and projective over $k ; Y$ is a smooth irreducible hypersurface of $X$ not contained in $\operatorname{BL}(P)$ (with the ideal sheaf $I_{Y}$ ); and for any $y \in \operatorname{BL}(\hat{P})$ and $U \subset X$ an affine connected neighbourhood of $y$, the ideal $I_{Y}(U) R(U, y)$ is a prime ideal of $R(U, y)$ where $R(U, y)$ is the integral closure of ${\widehat{\mathcal{O}_{X}(U)}}^{y}$ in $P(y, U)$.

Remark 8.6. Note that under the Hypothesis 8.5 on $(X, P)$ and $Y$, for $y \in Y$ of codimension at least one $P_{\mid Y}(y)$ is the fraction field of $R(U, y) /\left(I_{Y}(U)\right)$ where $R(U, y)$ is the integral closure of ${\widehat{\mathcal{O}_{X}(U)}}^{y}$ in $P(y, U)$. The hypothesis can be thought of as a variant of Hilbert irreducibility theorem as well in local setup.

Example. Let $X=\mathbb{P}_{k}^{n}$ with homogeneous coordinates $x_{0}, \ldots x_{n}, f: Z \rightarrow X$ be the normalization of $X$ in $k\left(x_{1} / x_{0}, \ldots x_{n} / x_{0}\right)[z] /\left(z^{p}-z-x_{n} / x_{0}\right)$ and the branch data $P=B_{f}$. Let $1 \leq i \leq n-1$ be an integer, $Y$ be the hypersurface in $\mathbb{P}^{n}$ defined by $x_{i}=0$. Then it is easy to check that $(X, P)$ and $Y$ satisfy Hypothesis 8.5

Let the formal orbifold $(X, P)$ and the hypersurface $Y$ satisfy Hypothesis 8.5 For a closed point $y \in \widehat{X}_{Y}$ and an affine neighbourhood $\widehat{U}_{y} \subset \widehat{X}_{Y}$ of $y$, define $A\left(\widehat{U}_{y}\right)$ to be the integral closure of $\mathcal{O}_{\widehat{X}_{Y}}\left(\widehat{U}_{y}\right)$ in $P\left(\eta_{Y}, U_{y}\right)$ where $U_{y} \subset X$ is an affine neighbourhood of $y$ viewed as a point in $X$ whose completion along $Y \cap U_{y}$ is $\widehat{U}_{y}$. Let $\mathcal{A}_{\widehat{U}_{y}}$ be the sheaf of algebras on $\widehat{U}_{y}$ associated to $A\left(\widehat{U}_{y}\right)$. Note that $\mathcal{U}=\left\{\widehat{U}_{y}: y \in Y\right.$ closed point $\}$ is an open cover of $\widehat{X}_{Y}$ and $P$ being branch data there are natural isomorphisms between $\mathcal{A}_{U_{1}}\left(U_{1} \cap U_{2}\right)$ and $\mathcal{A}_{U_{2}}\left(U_{1} \cap U_{2}\right)$ for all $U_{1}, U_{2} \in \mathcal{U}$ and they behave well with triple intersections. Hence there is a sheaf of algebras $\mathcal{A}$ on $\widehat{X}_{Y}$ whose restriction to $\widehat{U}_{y}$ is $\mathcal{A}_{\widehat{U}_{y}}$. Note that $\mathcal{A}$ is a sheaf of finite coherent $\mathcal{O}_{\widehat{X}_{Y}}$-algebras.

Definition 8.7. Let $\widehat{Z}=\operatorname{Spf}(\mathcal{A})$ be the formal scheme associated to $\mathcal{A}$ and $c$ : $\widehat{Z} \rightarrow \widehat{X}_{Y}$ be the structure morphism. We shall call $\widehat{Z}$ to be the normalization of $\widehat{X}_{Y}$ in $P$. Note that $\widehat{Z}$ is normal and $c$ is a finite morphism.

Proposition 8.8. Let $(X, P)$ and $Y$ satisfy the Hypothesis 8.5. Let $F$ be the residue field of the local ring ${\overline{\widehat{\mathcal{O}}_{X, \eta_{Y}}}}^{P\left(\eta_{Y}\right)}$ where $\eta_{Y}$ is the generic point of $Y$. Then $F / k(Y)$ is a Galois extension with $\operatorname{Gal}(F / k(Y))=\operatorname{Gal}\left(P\left(\eta_{Y}\right) / \mathcal{K}_{X, \eta_{Y}}\right)$. Let $\widehat{Z}$ be the normalization of $\widehat{X}_{Y}$ in $P$ (in the sense of the above definition) and $c_{0}: Z_{0} \rightarrow Y$ be the normalized pull-back of $c: \widehat{Z} \rightarrow \widehat{X}_{Y}$ along $Y \rightarrow \widehat{X}_{Y}$ then $Z_{0}$ is the normalization of $Y$ in $F$. Moreover, the morphisms $(\widehat{Z}, O) \rightarrow\left(\widehat{X}_{Y}, \hat{P}\right)$ and $\left(Z_{0}, O\right) \rightarrow\left(Y, P_{\mid Y}\right)$ are étale morphisms.

Proof. Note that $P\left(\eta_{Y}\right) / \mathcal{K}_{X, \eta_{Y}}$ is a Galois extension and the point $\eta_{Y}$ is not in $\mathrm{BL}(P)$ so the residue field extension $F / k(Y)$ is Galois with an epimorphism from $\operatorname{Gal}\left(P\left(\eta_{Y}\right) / \mathcal{K}_{X, \eta_{Y}}\right) \rightarrow \operatorname{Gal}(F / k(Y))$. Let $I_{Y}$ be the sheaf of ideals on $X$ defining $Y$ 
and $I_{Y, \eta_{Y}}$ be the prime ideal of $\widehat{\mathcal{O}}_{X, \eta_{Y}}$ generated by the stalk of $I_{Y}$ at $\eta_{Y}$. Note that the Hypothesis 8.5 ensures that there is only one prime ideal lying above $I_{Y, \eta_{Y}}$ in $\widehat{\widehat{\mathcal{O}}}_{X, \eta_{Y}} P\left(\eta_{Y}\right)$ and $I_{Y, \eta_{Y}}$ is unbranched in the extension $\widehat{\mathcal{O}}_{X, \eta_{Y}} P\left(\eta_{Y}\right) / \widehat{\mathcal{O}}_{X, \eta_{Y}}$. Hence the degree of the residue extension $F / k(Y)$ is same as that of $P\left(\eta_{Y}\right) / \mathcal{K}_{X, \eta_{Y}}$. Hence the two Galois groups are isomorphic.

Note that $\widehat{Z} \rightarrow \widehat{X}_{Y}$ is generically étale and $\eta_{Y}$ is unramified and non-split in this cover. Hence the closed fiber, i.e., the pull-back of the formal scheme $\widehat{Z}$ along $Y \rightarrow$ $\widehat{X}_{Y}$ is an integral scheme and its function field is $F$. Hence $Z_{0}$ is the normalization of the closed fiber of $\widehat{Z}$. Since the closed fiber of $Z$ is a finite cover of $Y, Z_{0}$ is the normalization of $Y$ in $F$.

For a point $z$ of $\widehat{Z}$ of codimesion at least one in the closed fiber, let $x$ denote its image in $\widehat{X}_{Y}$. Then $x$ is of codimension at least one in $Y$. Note that $\mathcal{K}_{Z, z}=$ $\mathcal{K}_{\widehat{Z}, z}$ is the fraction field of the completion of ${\overline{\mathcal{O}} \widehat{X}_{Y}, x} P\left(\eta_{Y}\right)$ along the prime ideal $I_{z}$ corresponding to the point $z$. But this ring is same as the integral closure of $\widehat{\mathcal{O}}_{\widehat{X}_{Y}, x}$ in $P\left(\eta_{Y}\right) \mathcal{K}_{X, x}$. Hence $\mathcal{K}_{Z, z}=P\left(\eta_{Y}\right) \mathcal{K}_{X, x}=P(x)$. This proves $(Z, O) \rightarrow\left(\widehat{X}_{Y}, P\right)$ is étale.

Let $z_{0} \in Z_{0}$ be a point of codimension at least one and $y \in Y$ be its image. Then $\mathcal{K}_{Z_{0}, z_{0}}=\mathcal{K}_{Y, y} F$ since $F$ is the function field of $Z_{0}$. But $F={\widehat{\widehat{\mathcal{O}}_{X, \eta_{Y}}}}^{P\left(\eta_{Y}\right)} /\left(I_{Y, \eta_{Y}}\right)$ as the Hypothesis 8.5 implies that $I_{Y, \eta_{Y}}$ generates the maximal ideal of $\frac{\widehat{\mathcal{O}}_{X, \eta_{Y}}}{P\left(\eta_{Y}\right)}$. Now,

$$
\begin{aligned}
\mathcal{K}_{Z_{0}, z_{0}} & =\mathcal{K}_{Y, y}{\overline{\widehat{\mathcal{O}}_{X, \eta_{Y}}}}^{P\left(\eta_{Y}\right)} /\left(I_{Y, \eta_{Y}}\right) \\
& =\mathcal{K}_{Y, y} \widehat{\mathcal{O}}_{\widehat{Z}, \eta_{Y}} /\left(I_{Y, \eta_{Y}}\right) \\
& =\mathcal{K}_{Y, y} \mathrm{QF}\left(\mathcal{O}_{\widehat{V}} /\left(I_{Y}\right)\right) \text { where } \widehat{V} \subset \widehat{Z} \text { is an affine open neighbourhood of } \eta_{Y} \\
& =\mathrm{QF}\left(\widehat{\mathcal{O}}_{\widehat{V}, z} /\left(I_{Y, z}\right)\right) \text { where } z \text { is the image of } z_{0} \text { in } \widehat{Z} \\
& =\operatorname{QF}\left(\widehat{\widehat{\mathcal{O}}}_{\widehat{X}_{Y}, y} P(y)\right. \\
& =P_{Y Y}(y)
\end{aligned}
$$

Hence $\left(Z_{0}, O\right) \rightarrow\left(Y, P_{\mid Y}\right)$ is étale.

Now we proceed following the strategy in GM, Section 4.3] to show that the functor $\operatorname{Cov}\left(\widehat{X}_{Y}, \hat{P}\right) \rightarrow \operatorname{Cov}\left(Y, P_{\mid Y}\right)$ is an equivalence of category.

Proposition 8.9. Under the Hypothesis 8.5, the functor $\operatorname{Cov}\left(\widehat{X}_{Y}, \hat{P}\right) \rightarrow \operatorname{Cov}\left(Y, P_{\mid Y}\right)$ is faithful.

Proof. Let $f, g: \mathcal{U} \rightarrow \mathcal{V}$ be morphisms in $\operatorname{Cov}\left(\widehat{X}_{Y}, \hat{P}\right)$ and $u: \mathcal{U} \rightarrow\left(\widehat{X}_{Y}, \hat{P}\right)$ and $v: \mathcal{V} \rightarrow\left(\widehat{X}_{Y}, \hat{P}\right)$ be étale morphism. Assume $f_{0}=g_{0}: \mathcal{U}_{0} \rightarrow \mathcal{V}_{0}$ where $\mathcal{U}_{0}$ and $\mathcal{V}_{0}$ are the normalized pullback of $\mathcal{U}$ and $\mathcal{V}$ along $Y \rightarrow \widehat{X}_{Y}$. We wish to show $f=g$. Let $f^{*}$ and $g^{*}$ from $\mathcal{U}^{*} \rightarrow \mathcal{V}^{*}$ be the pullback of $f$ and $g$ respectively along $(Z, O) \rightarrow\left(\widehat{X}_{Y}, \hat{P}\right)$ of the above proposition. Note that the branch data on $\mathcal{U}^{*}$ and $\mathcal{V}^{*}$ are trivial (as $(Z, O)$ has trivial branch data and $u, v$ are étale). Also $\mathcal{U}^{*} \rightarrow(Z, O)$ and $\mathcal{V}^{*} \rightarrow(Z, O)$ are étale morphisms. Let $\left(f_{0}\right)^{*}=\left(g_{0}\right)^{*}$ be the morphism $\mathcal{U}_{0}^{*} \rightarrow \mathcal{V}_{0}^{*}$ where $\mathcal{U}_{0}^{*}$ and $\mathcal{V}_{0}^{*}$ are the normalized pull-backs of $\mathcal{U}_{0}$ and $\mathcal{V}_{0}$ along $Z_{0} \rightarrow Y$. Observe that the normalization of the closed fibers of $\mathcal{U}^{*}$ and $\mathcal{V}^{*}$ 
are same as $\mathcal{U}_{0}^{*}$ and $\mathcal{V}_{0}^{*}$ respectively. Also note that $\left(f^{*}\right)_{0}=\left(f_{0}\right)^{*}=\left(g_{0}\right)^{*}=\left(g^{*}\right)_{0}$ where $\left(f^{*}\right)_{0}$ and $\left(g^{*}\right)_{0}$ are morphisms between the normalization of the closed fibers of $\mathcal{U}^{*}$ and $\mathcal{V}^{*}$. Hence $f^{*}=g^{*}$ by [GM] étale case]. Now away from the branch locus $Z \rightarrow \widehat{X}_{Y}$ is étale, hence by applying flat descent on this locus there exists an open dense $W \subset \widehat{X}_{Y}$ such that $f=g$ when restricted to $u^{-1}(W)$. But $u^{-1}(W)$ is dense in $\mathcal{U}$, hence $f=g$.

Proposition 8.10. Under the Hypothesis 8.5 , the functor $\operatorname{Cov}\left(\widehat{X}_{Y}, \hat{P}\right) \rightarrow \operatorname{Cov}\left(Y, P_{\mid Y}\right)$ is fully faithful.

Proof. Again we use the argument similar to [GM, 4.3.6]. Let $Z \rightarrow \widehat{X}_{Y}$ and $Z_{0} \rightarrow Y$ be the covers in Proposition 8.8 and $G$ denote their common Galois group. Let $u: \mathcal{U} \rightarrow\left(\widehat{X}_{Y}, \hat{P}\right)$ and $v: \mathcal{V} \rightarrow\left(\widehat{X}_{Y}, \hat{P}\right)$ be étale covers and $g_{0}: \mathcal{U}_{0} \rightarrow \mathcal{V}_{0}$ be a morphism in $\operatorname{Cov}\left(Y, P_{\mid Y}\right)$. Let $g_{0}^{*}: \mathcal{U}_{0}^{*} \rightarrow \mathcal{V}_{0}^{*}$ be the morphism obtained by pulling back $g_{0}$ to $Z_{0}$. Now $g_{0}^{*}$ is étale and the branch data on $\mathcal{U}_{0}^{*}$ and $\mathcal{V}_{0}^{*}$ are trivial. Rest of the proof is same as [GM, 4.3.6] with $\mu_{n}$ replaced by $G$.

Theorem 8.11. Under the Hypothesis [8.5, the functor $\operatorname{Cov}\left(\widehat{X}_{Y}, \hat{P}\right) \rightarrow \operatorname{Cov}\left(Y, P_{\mid Y}\right)$ is an equivalence.

Proof. Here also the proof is same as in [GM, 4.3.7] with appropriate modifications, i.e. the Kummer covers being replaced by the covers $Z \rightarrow \widehat{X}_{Y}$ and $Z_{0} \rightarrow Y$ of Proposition 8.8

8.2. The functor $\operatorname{Cov}(X, P)$ to $\operatorname{Cov}\left(\widehat{X}_{Y}, \hat{P}\right)$. We need the following lemma.

Lemma 8.12. Let $R$ be a ring and $M, N$ be $R$-modules, let $r_{1}, r_{2}$ be a regular sequence on $N$ then $r_{1}, r_{2}$ is a regular sequence on $\operatorname{Hom}_{R}(M, N)$. In particular, if depth of $N$ is at least 2 then depth of $\operatorname{Hom}_{R}(M, N)$ is also at least 2.

Proof. Let $f \in \operatorname{Hom}_{R}(M, N)$ and $r_{1} f=0$ then $r_{1} f(m)=0$ for all $m \in M$. But $r_{1}$ is regular on $N$ implies $f(m)=0$ for all $m \in M$, i.e. $f=0$. Hence $r_{1}$ is a nonzero divisor on $\operatorname{Hom}_{R}(M, N)$.

Let $\bar{g} \in \operatorname{Hom}_{R}(M, N) / r_{1} \operatorname{Hom}_{R}(M, N)$ be the image of $g \in \operatorname{Hom}_{R}(M, N)$. We need to show that if $r_{2} \bar{g}=0$ then $g \in r_{1} \operatorname{Hom}_{R}(M, N)$. Note that $r_{2} \bar{g}=\overline{r_{2} g}=0$ implies $r_{2} g \in r_{1} \operatorname{Hom}_{R}(M, N)$. Hence there exists $f \in \operatorname{Hom}_{R}(M, N)$ such that $r_{2} g(m)=r_{1} f(m)$ for all $m \in M$. Hence $g(m) \in r_{1} N$ for all $m \in M$. But $r_{1}, r_{2}$ is a regular sequence on $N$, so $g(m) \in r_{1} N$ for all $m \in M$. Hence $g \in$ $r_{1} \operatorname{Hom}_{R}(M, N)$.

The pullback along the natural morphism $\widehat{X}_{Y} \rightarrow X$ defines a functor from $\operatorname{Cov}(X, P) \rightarrow \operatorname{Cov}\left(\widehat{X}_{Y}, \hat{P}\right)$.

Proposition 8.13. Let $(X, P)$ be a projective formal orbifold over $k$ of dimension at least two, $Y$ be a normal hypersurface of $X$ which intersects $\mathrm{BL}(P)$ transversally and the divisor $[Y]$ is a very ample divisor on $X$. Then the functor $\operatorname{Cov}(X, P) \rightarrow$ $\operatorname{Cov}\left(\widehat{X}_{Y}, \hat{P}\right)$ is fully faithful.

Proof. Let $u: \mathcal{Z} \rightarrow(X, P)$ and $v: \mathcal{W} \rightarrow(X, P)$ be in $\operatorname{Cov}(X, P)$. It is enough to show that $\operatorname{Hom}_{X}(Z, W) \cong \operatorname{Hom}_{\widehat{X}_{Y}}\left(Z_{Y}, W_{Y}\right)$ where $Z_{Y}$ and $W_{Y}$ are the pullback of $u$ and $v$ to $\widehat{X}_{Y}$. Note that $Z_{Y}$ and $W_{Y}$ are normal. Note that $\operatorname{Hom}_{X}(Z, W)$ are same as homomorphisms between sheaf of $\mathcal{O}_{X}$-algebras $v_{*} \mathcal{O}_{W} \rightarrow u_{*} \mathcal{O}_{Z}$ as $u, v$ and every $X$-morphism from $Z$ to $W$ are finite (and hence affine). Since 
$Z$ and $W$ are normal of dimension at least $2, u_{*} O_{Z}$ has depth at least 2 and hence by Lemma 8.12 coherent sheaf of $\mathcal{O}_{X}$-modules $\underline{\operatorname{Hom}}_{\mathcal{O}_{X}}\left(v_{*} \mathcal{O}_{W}, u_{*} \mathcal{O}_{Z}\right)$ has depth at least 2 (here we consider all $\mathcal{O}_{X}$-module homomorphism and not just algebra homomorphism). Using [SGA2, Chapter XII, Corollary 2.2], we obtain that $\operatorname{Hom}_{\mathcal{O}_{X}}\left(v_{*} \mathcal{O}_{W}, u_{*} \mathcal{O}_{Z}\right) \cong \operatorname{Hom}_{\mathcal{O}_{\bar{X}_{Y}}}\left(\widehat{v_{*} \mathcal{O}_{W}}, \widehat{u_{*} \mathcal{O}_{Z}}\right)$ but the right side equals $\operatorname{Hom}_{\widehat{\mathcal{X}}_{\widehat{X}_{Y}}}\left(v_{*} \mathcal{O}_{\widehat{W}_{Y}}, u_{*} \mathcal{O}_{\widehat{Z}_{Y}}\right)$. Also since $\widehat{X}_{Y} \rightarrow X$ is flat Hom behaves well with tensor product. So the bijection takes an algebra homomorphism between $v_{*} \mathcal{O}_{W}$ and $u_{*} \mathcal{O}_{Z}$ to an algebra homomorphism between $v_{*} \mathcal{O}_{\widehat{W}_{Y}}$ and $u_{*} \mathcal{O}_{\widehat{Z}_{Y}}$. Hence the functor is fully faithful.

In fact like in [SGA2, Chapter XII], even the following is true.

Proposition 8.14. Let $U$ be an open neighborhood of $Y$. Then the functors $\operatorname{Cov}(X, P) \rightarrow \operatorname{Cov}\left(U, P_{\mid U}\right)$ and $\operatorname{Cov}\left(U, P_{\mid U}\right) \rightarrow \operatorname{Cov}\left(\widehat{X}_{Y}, \hat{P}\right)$ are fully faithful.

Proof. Note that the composition of these two functors is the functor in the above proposition. We use the notation of the above proof. Since $\underline{\operatorname{Hom}}_{\mathcal{O}_{X}}\left(v_{*} \mathcal{O}_{W}, u_{*} \mathcal{O}_{Z}\right)$ has depth at least 2 (Lemma 8.12), it is torsion free. Hence the homomorphisms $\operatorname{Hom}_{\mathcal{O}_{X}}\left(v_{*} \mathcal{O}_{W}, u_{*} \mathcal{O}_{Z}\right) \rightarrow \operatorname{Hom}_{\mathcal{O}_{U}}\left(v_{*} \mathcal{O}_{W_{U}}, u_{*} \mathcal{O}_{Z_{U}}\right)$ and $\operatorname{Hom}_{\mathcal{O}_{U}}\left(v_{*} \mathcal{O}_{W_{U}}, u_{*} \mathcal{O}_{Z_{U}}\right) \rightarrow$ $\operatorname{Hom}_{\widehat{\mathcal{X}}_{\widehat{X}_{Y}}}\left(v_{*} \mathcal{O}_{\widehat{W}_{Y}}, u_{*} \mathcal{O}_{\widehat{Z}_{Y}}\right)$ are injective (for the injectivity of the second map use the argument in [SGA2, Chapter XII, Corollary 2.4]). By the above proposition the composition is an isomorphism hence the two maps are isomorphisms.

As a consequence of Theorem 8.11 and Proposition 8.13 we get the following corollary.

Corollary 8.15. Let $(X, P)$ be a smooth irreducible projective formal orbifold over a perfect field $k$ of dimension at least two. The functor from $\operatorname{Cov}(X, P) \rightarrow$ $\operatorname{Cov}\left(Y, P_{\mid Y}\right)$ is fully faithful if $Y$ satisfies Hypothesis 8.5. In particular the map natural map $\pi_{1}\left(Y, P_{\mid Y}\right) \rightarrow \pi_{1}(X, P)$ is an epimorphism.

\section{LOCALLY CONSTANT $l$-ADiC SHEAF}

Let $X$ be a normal geometrically connected variety over a field $k$ of characteristic $p$. Recall that given a finite locally constant sheaf $\mathcal{F}$ on $X$ with stalk $A$ it corresponds to a representation $\rho_{\mathcal{F}}: \pi_{1}(X, x) \rightarrow \operatorname{Aut}(A)$ where $x$ is a geometric point of $X$. The converse also holds. This result and its lisse $l$-adic version hold for geometric formal orbifolds as well.

Definition 9.1. Let $l \neq p$ be a prime number. An $l$-adic sheaf is a locally constant sheaf of $R$-modules where $R$ is the integral closure of $\mathbb{Z}_{l}$ in a finite extension $K$ of $\mathbb{Q}_{l}$. Note that $R$ is a complete DVR and let $\mathfrak{m}$ denote its maximal ideal. A lisse $l$ adic sheaf of $R$-modules is a compatible system of locally constant sheaves $\left(\mathcal{F}_{n}\right)_{n \geq 0}$ where $\mathcal{F}_{n}$ is a locally constant $R_{n}=R / \mathfrak{m}^{n+1}$-module and $\mathcal{F}_{n+1} \otimes_{R_{n+1}} R_{n} \cong \mathcal{F}_{n}$. The morphisms in this category are defined to be compatible system of morphisms. For more details see [FK, Chapter 1, Section 12]. The category of lisse $K$-sheaf consist of lisse $l$-adic sheaf of $R$-modules as objects and the morphisms are given by $\operatorname{Hom}_{K}\left(\mathcal{F}, \mathcal{F}^{\prime}\right):=\operatorname{Hom}\left(\mathcal{F}^{\prime}, \mathcal{F}^{\prime}\right) \otimes_{R} K$.

Proposition 9.2. Let $\mathcal{F}$ be a finite locally constant sheaf with stalk $A$ on a geometric formal orbifold $\mathcal{X}=(X, P)$. Let $X^{\circ}=X \backslash \mathrm{BL}(P)$. The restriction 
$\mathcal{F}^{o}=\mathcal{F}_{\mid X^{\circ}}$ is a locally constant sheaf and hence $\mathcal{F}^{o}$ correspond to a representation $\rho: \pi_{1}\left(X^{o}, x\right) \rightarrow \operatorname{Aut}\left(\mathcal{F}_{x}\right)$ where $x \in X^{o}$ is a geometric point. Then $\rho$ factors through $\pi_{1}(\mathcal{X}, x)$ to give a continuous representation $\bar{\rho}: \pi_{1}(\mathcal{X}, x) \rightarrow \operatorname{Aut}\left(\mathcal{F}_{x}\right)$. Conversely given a continuous representation $\bar{\rho}: \pi_{1}(\mathcal{X}, x) \rightarrow \operatorname{Aut}(A)$ it induces a finite locally constant sheaf $\mathcal{F}$ on $\mathcal{X}$ with stalk $A$. And the two functors are inverse to each other.

Proof. Since $\mathcal{X}=(X, P)$ is a geometric formal orbifold and $X$ is geometrically connected there exists a finite Galois étale cover of formal orbifolds $h:(Z, O) \rightarrow \mathcal{X}$, with $Z$ geometrically connected. Since $\mathcal{F}$ is a locally constant sheaf on $\mathcal{X}$ so is $h^{*} \mathcal{F}$. The étale site on $(Z, O)$ is the usual étale site on $Z$. Hence there exists an étale Galois cover $g: Y \rightarrow Z$ with $Y$ connected such that the pull back $g^{*} h^{*} \mathcal{F}$ is constant. Let $f=h \circ g$ and passing to Galois closure we may assume $f$ is a Galois cover. Let $Y^{o}=f^{-1}\left(X^{o}\right)$. The restriction of $f^{*} \mathcal{F}$ to $Y^{o}$ is constant. Hence $\rho$ factors through $\pi_{1}\left(X^{o}\right) / \pi_{1}\left(Y^{o}\right)$ which is same as $\pi_{1}(\mathcal{X}) / \pi_{1}(Y)$. Hence $\rho$ factors through $\pi_{1}(\mathcal{X})$.

Conversely, let $\bar{\rho}: \pi_{1}(\mathcal{X}, x) \rightarrow A u t(A)$ be a continuous representation. Since $A$ is finite, $\bar{\rho}$ factors through a finite quotient of $\pi_{1}(\mathcal{X})$ say $G$. This finite quotient correspond to a $G$-Galois étale cover $f:(Y, Q) \rightarrow \mathcal{X}$ and we may assume $Q$ is the trivial branch data. Consider the constant sheaf $A_{Y}$ on $Y$. The group homomorphism $G \rightarrow A u t(A)$ makes $A_{Y}$ into a $G$-sheaf. In other words the action of $G$ on $Y$ lifts to $A_{Y}$ via the group homomorphism $G \rightarrow A_{Y}$. Then $f_{*} A_{Y}$ is a sheaf on $\mathcal{X}$ with a $G$-action. Set $\mathcal{F}:=\left(f_{*} A_{Y}\right)^{G}$. Then $\mathcal{F}$ is a locally constant sheaf on $\mathcal{X}$.

The two functors are inverse to each other can be seen by using the corresponding result in the variety case.

Corollary 9.3. The category of lisse l-adic sheaf of $K$-modules on $\mathcal{X}$ is equivalent to the category of continuous representations $\rho: \pi_{1}(\mathcal{X}) \rightarrow \mathrm{GL}_{N}(K)$ for any $K / \mathbb{Q}_{l}$ a finite field extension.

Proof. In view of the above proposition, the proof is same as the case of normal varieties.

We collect some definitions on tameness and bounded ramification of $l$-adic sheaves from [Dri], Esn] and [EKe.

Definition 9.4. Let $X$ be a normal geometrically connected variety over $k$ and $\mathcal{F}$ a locally constant sheaf on $X$.

(1) If $\operatorname{dim}(X)=1$ then $\mathcal{F}$ is called tame if $\rho_{\mathcal{F}}$ factors through the tame quotient of the fundamental group $\pi_{1}^{t}(X, x)$.

(2) For $X$ of arbitrary dimension, $\mathcal{F}$ is tame if for any geometrically irreducible curve $C$ in $X$ with normalization $\tilde{C}$, the pull back of $\mathcal{F}$ to $\tilde{C}$ is tame.

(3) A lisse $l$-adic sheaf $\mathcal{F}=\left(\mathcal{F}_{n}\right)$ on $X$ is called tame if for all $n, \mathcal{F}_{n}$ are tame on $X$.

(4) A lisse $l$-adic sheaf $\mathcal{F}$ on $X$ is said to have ramification bounded by $f$ : $Y \rightarrow X$ a finite dominant morphism of normal varieties if $f^{*} \mathcal{F}$ is tame on $Y$.

The following notions of tameness of a branch data are defined in analogy with KSc.

Definition 9.5. Let $P$ be a branch data on a normal variety $X$. 
(1) For a point $x \in X$ of codimension at least one, $P$ is called numerically-tame at $x$ if the inertia group $I\left(P(x) / \mathcal{K}_{X, x}\right)$ is of order prime to $p$. The branch data $P$ is called numerically-tame if $P$ is numerically tame for all points $x \in X$ of codimension at least one. It is enough to check numerically-tame for all closed points.

(2) For a closed point $x \in X, P$ is called curve-tame at $x$ if the following holds: For any codimension one prime ideal $\mathfrak{p}$ in $\widehat{\mathcal{O}}_{X, x}$ not in $\operatorname{BL}(P)$ and for any codimension one prime ideal $\mathfrak{q}$ in the integral closure $\mathcal{O}_{P(x)}$ of $\widehat{\mathcal{O}}_{X, x}$ in $P(x)$ lying above $\mathfrak{p}$, let $R$ and $S$ be the normalization of $\widehat{\mathcal{O}}_{X, x} / \mathfrak{p}$ and $\mathcal{O}_{P(x)} / \mathfrak{q}$ respectively. Then $S / R$ is at most tamely ramified extension. The branch data $P$ is called curve-tame if it is curve-tame for all closed points $x \in X$.

Proposition 9.6. Let $X$ be a proper normal variety over $k$ and $f: Y \rightarrow X$ be a Galois cover which is étale over $X^{\circ}$ an open subset of regular locus of $X$. The branch data $B_{f}$ is curve-tame iff $f$ is curve-tame.

Proof. Assume $f$ is curve-tame. Let $x \in X$ be a closed point. Let $\mathfrak{p}$ be a codimension one prime ideal of $\widehat{\mathcal{O}}_{X, x}$ not in $\operatorname{BL}\left(B_{f}\right)$ and $\mathfrak{q}$ be a codimension one prime ideal in the integral closure $\mathcal{O}_{B_{f}(x)}$ of $\mathcal{O}_{X, x}$ in $B_{f}(x)$ lying above $\mathfrak{p}$. Then $\mathfrak{p}$ defines an integral curve $C$ in $X$ passing through $x$ which intersects $X^{o}$ and $C$ is unibranched at $x$. Let $\tilde{C}$ be the normalization of $C$ and $\tilde{x}$ be the point in $\tilde{C}$ lying above $x \in C$.

Let $Y_{C}=\widetilde{Y \times_{X}} \tilde{C}$ be the normalized fiber product. Since $f$ is curve-tame, $Y_{C} \rightarrow \tilde{C}$ is at most tamely ramified. Note that there exists $y \in Y$ lying above $x$ such that $\widehat{\mathcal{O}}_{Y, y}=\mathcal{O}_{B_{f}(x)}$. The normalization $S$ of $\widehat{\mathcal{O}}_{Y, y} / \mathfrak{q}$ is same as $\widehat{\mathcal{O}}_{D, z}$ where $D$ is a connected component of $Y_{C}$ and $z$ is a point on $Y_{C}$ lying above $(y, \tilde{x})$ in the fiber product. Since $Y_{C} \rightarrow \tilde{C}$ is at most tamely ramified, the extension $\widehat{\mathcal{O}}_{D, z} / \widehat{\mathcal{O}}_{\tilde{C}, \tilde{x}}$ is at most tamely ramified. But $\widehat{\mathcal{O}}_{\tilde{C}, \tilde{x}}$ is the normalization of $\widehat{\mathcal{O}}_{X, x} / \mathfrak{p}$. Hence $B_{f}$ is curve tame at $x$.

The converse is also a translation between algebra and geometry and follows after noting that the tameness of ramification does not change when passed to completion.

The following result is a direct consequence of [KSc, Theorem 5.3, Theorem 5.4].

Proposition 9.7. Let $(X, P)$ be a proper formal orbifold with $P$ a numericallytame branch data and $X^{o}=X \backslash \mathrm{BL}(P)$ regular. Let $f:(Y, O) \rightarrow(X, P)$ be an étale Galois cover. Then $f: Y \rightarrow X$ is curve-tame. In other words $P$ is curve tame. The converse hold if $X$ is regular and $\mathrm{BL}(P)$ is a normal crossing divisor.

Proof. The cover $f:(Y, O) \rightarrow(X, P)$ is an étale cover implies that $f: Y \rightarrow$ $X$ is étale over $X^{o}$ and numerically tamely ramified cover w.r.t to the normal compactification $X$. Now the result follows from $[\mathrm{KSc}$, Theorem 5.3, Theorem $5.4]$

Proposition 9.8. Let $X$ be a normal connected proper variety and $X^{o}$ be an open subset of the regular locus of $X$. Let $f: Y \rightarrow X$ be a Galois cover étale over $X^{\circ}$. Any finite locally constant $l$-adic sheaf $\mathcal{F}$ on a geometric formal orbifold $(X, P)$ where $P \geq B_{f}$ with $\mathrm{BL}(P) \cap X^{o}=\emptyset$ and $P(x) / B_{f}(x)$ at most curve tamely ramified for all closed points $x \in X$ restricts to a locally constant finite l-adic sheaf on $X^{o}$ whose ramification is bounded by $f$. 
Proof. Let $\mathcal{F}^{o}=\left.\mathcal{F}\right|_{X^{o}}$. Let $g:(Z, O) \rightarrow(X, P)$ be an étale cover such that $g^{*} \mathcal{F}$ is a constant sheaf. Let $(W, O)$ be the fiber product of $g$ and $f:\left(Y, f^{*} P\right) \rightarrow(X, P)$ and $h:(W, O) \rightarrow\left(Y, f^{*} P\right)$ be the projection map. Note that $h$ is étale and $f^{*} P$ is a curve-tame branch data since $P(x) / B_{f}(x)$ is curve-tamely ramified for all $x \in X$. So $h: W \rightarrow Y$ is a curve-tamely ramified cover. Now $f^{*} \mathcal{F}^{o}$ is a locally constant sheaf on $Y^{o}=f^{-1}\left(X^{o}\right)$ and $h^{*} f^{*} \mathcal{F}^{o}=g^{*} \mathcal{F}^{o}$ is a constant sheaf. Hence $f^{*} \mathcal{F}^{o}$ is tame $l$-adic sheaf.

The converse also holds.

Proposition 9.9. Let $X$ be a normal connected proper variety and $X^{\circ}$ be an open subset of the regular locus of $X$. Let $\mathcal{F}^{o}$ be a locally constant finite l-adic sheaf on $X^{o}$ whose ramification is bounded by a Galois cover $f: Y \rightarrow X$ which is étale over $X^{o}$. Let $B_{f}$ be the branch data on $X$ associated to $f$. Then $\mathcal{F}^{\circ}$ extends to a finite locally constant l-adic sheaf $\mathcal{F}$ on $(X, P)$ for some geometric branch data $P \geq B_{f}$ with $\mathrm{BL}(P) \cap X^{o}=\emptyset$ and $P(x) / B_{f}(x)$ at most curve-tamely ramified for all closed points $x \in X$.

Proof. By Proposition 9.2 a finite locally constant $l$-adic sheaf $\mathcal{F}^{o}$ on $X^{o}$ correspond to a continuous representation $\rho: \pi_{1}\left(X^{o}, x\right) \rightarrow \operatorname{Aut}\left(\mathcal{F}_{x}^{o}\right)$. Since ramification of $\mathcal{F}^{o}$ is bounded by $f, f^{*} \mathcal{F}^{o}$ is tame finite locally constant sheaf. So there exists a finite curve-tamely ramified Galois cover $h^{\prime}: Y^{\prime} \rightarrow Y$ étale over $X^{o}$ such that $h^{*} f^{*} \mathcal{F}^{o}$ is a constant sheaf. Let $g: Z \rightarrow X$ be the Galois closure of $f \circ h^{\prime}: Y^{\prime} \rightarrow X$. Then $g$ is étale over $X^{o}$ and the natural map $h: Z \rightarrow Y$ is curve tamely ramified (being compositum of covers isomorphic to $h^{\prime}: Y^{\prime} \rightarrow Y$ which which is curvetamely ramified). Let $P=B_{g}$ then $g:(Z, O) \rightarrow(X, P)$ is étale, $P$ is a geometric branch data, $\mathrm{BL}(P) \cap X^{o}=\emptyset, P \geq B_{f}$ and $\rho$ factors through $\pi_{1}((X, P), x)$. Let $\mathcal{F}$ be the extension of $\mathcal{F}^{o}$ to $(X, P)$ (obtained from Proposition 9.2). The extension $\mathcal{K}_{Z, z} / \mathcal{K}_{Y, h(z)}$ is at most curve-tamely ramified for all closed points $z \in Z$ since $h$ is curve-tamely ramified. But $\mathcal{K}_{Z, z}=P(g(z))$ and $\mathcal{K}_{Y, h(z)}=B_{f}(f(h(z)))=B_{f}(g(z))$, so $P(x) / B_{f}(x)$ is at most curve-tamely ramified for all closed points $x \in X$.

Corollary 9.10. Let $\mathcal{F}^{o}$ be a lisse l-adic sheaf on $X^{o}$. Let $X$ be a normal compactification of $X^{o}$. For $i=1,2$ let $f_{i}: Y_{i} \rightarrow X$ be Galois covers of normal varieties étale over $X^{o}$ such that $Q_{1} B_{f_{1}}=Q_{2} B_{f_{2}}$ where $Q_{1}$ and $Q_{2}$ are curve-tame branch data on $X$ with branch locus disjoint with $X^{\circ}$. Then the ramification of $\mathcal{F}^{o}$ is bounded by $f_{1}$ iff it is bounded by $f_{2}$.

Proof. Let $\mathcal{F}^{o}=\left(\mathcal{F}_{n}^{o}\right)_{n \geq 0}$ where $\mathcal{F}_{n}^{o}$ are compatible finite locally constant $l$-adic sheaves. For $i=1,2$, by the above proposition the ramification of $\mathcal{F}_{n}^{o}$ is bounded by $f_{i}$ iff $\mathcal{F}_{n}^{o}$ extends to a locally constant sheaf on $(X, P)$ for some $P \geq B_{f_{i}}$ with $\mathrm{BL}(P) \cap X^{o}=$ and $P(x) / B_{f_{i}}(x)$ at most tamely ramified for all $x \in X$. Since $Q_{1}$ and $Q_{2}$ are tame branch data on $X$, for $i=1,2, P(x) Q_{i}(x) / B_{f_{i}}(x) Q_{i}(x)$ is at most tamely ramified iff $P(x) / B_{f_{i}}(x)$ is at most tamely ramified for all $x \in X$. Since $Q_{1} B_{f_{1}}=Q_{2} B_{f_{2}}$ we obtain that $\mathcal{F}_{n}^{o}$ is bounded by $f_{1}$ iff it is bounded by $f_{2}$.

In particular the above means that the local property at the boundary of an étale morphism $f: Y^{o} \rightarrow X^{o}$ decides whether an $l$-adic lisse sheaf on $X^{o}$ has ramification bounded by $f$. 


\section{REFERENCES}

[Abh] S. S. Abhyankar Ramification theoretic methods in algebraic geometry, Annals of Mathematics Studies, no. 43 Princeton University Press, Princeton, N.J. 1959 ix+96 pp.

[Bou] Nicholas Bourbaki Commutative algebra. Chapters 1-7 Translated from the French. Reprint of the 1989 English translation. Elements of Mathematics (Berlin). Springer-Verlag, Berlin, 1998. xxiv $+625 \mathrm{pp}$.

[Coh] I. S. Cohen On the structure and ideal theory of complete local rings, Trans. Amer. Math. Soc. 59, (1946), 54-106.

[Dri] Vladimir Drinfeld On a conjecture of Deligne, Mosc. Math. J. 12 (2012), no. 3, 515-542, 668.

[Esn] Hélène Esnault A remark on Deligne's finiteness theorem, http://arxiv.org/abs/1602.09032 (preprint)

[EKe] Hélène Esnault and Moritz Kerz A finiteness theorem for Galois representations of function fields over finite fields (after Deligne), Acta Math. Vietnam. 37 (2012), no. 4, 531-562.

[EKi] Hélène Esnault and Lars Kindler Lefschetz theorems for tamely ramified coverings, Proc. Amer. Math. Soc. 144 (2016), no. 12, 5071-5080. 14E20 (14E22 14F35)

[FK] E. Freitag and R. Kiehl Etale cohomology and the Weil conjectures, Ergebn. Math. Grenzg., 3. Folge 13, Springer, 1988.

[EGA 1] Alexander Grothendieck Éléments de géométrie algébrique. I. Le langage des schémas Inst. Hautes Études Sci. Publ. Math. No. 4 1960, 228 pp.

[EGA 42] Alexander Grothendieck Éléments de géométrie algébrique. IV. Étude locale des schémas et des morphismes de schémas. II Inst. Hautes Études Sci. Publ. Math. No. 24 1965, $231 \mathrm{pp}$.

[GM] ega4.2 Alexander Grothendieck and Jacob P. Murre The tame fundamental group of a formal neighbourhood of a divisor with normal crossings on a scheme, Lecture notes in Mathematics.

[SGA1] Revêtements étales et groupe fondamental (SGA 1), Lecture Notes in Mathematics, Vol. 224, Springer-Verlag, Berlin-New York, 1971 (French). Séminaire de géométrie algébrique du Bois Marie 1960-61; Directed by A. Grothendieck, With two papers by M. Raynaud.

[SGA2] Alexander Grothendieck Cohomologie locale des faisceaux cohérents et théorèmes de Lefschetz locaux et globaux (SGA 2) Augmenté d'un exposé par Michèle Raynaud, Séminaire de Géométrie Algébrique du Bois-Marie, 1962, Advanced Studies in Pure Mathematics, Vol. 2. North-Holland Publishing Co., Amsterdam; Masson \& Cie, Éditeur, Paris, 1968. vii+287 pp.

[Kat] Kazuya Kato Swan conductors for characters of degree one in the imperfect residue field case, Comtemp. Math., 83 (1989), 101-131.

[KS1] Moritz Kerz and Shuji Saito Lefschetz theorem for abelian fundamental group with modulus, Algebra Number Theory 8 (2014), no. 3, 689-701.

[KS2] Moritz Kerz and Shuji Saito Chow group of 0-cycles with modulus and higher dimensional class field theory http://arxiv.org/abs/1304.4400 (preprint).

[KSc] Moritz Kerz and Alexander Schmidt On different notions of tameness in arithmetic geometry, Math. Annalen 346, Issue 3 (2010), 641-668.

[Kum] Manish Kumar Killing wild ramification, Israel J. Math. 199 (2014), no. 1, 421-431.

[KP] Manish Kumar and A.J. Parameswaran Formal orbifolds and orbifold bundles in positive characteristic, http://arxiv.org/abs/1512.03235 (preprint).

[Mat] Shigeki Matsuda On the Swan conductors in positive characteristic, Amer. J. Math., 119 (1997) 705-739.

[Mil] James Milne Étale cohomology Princeton University Press, Princeton, N.J., 1980.

[SP] Stacks Project, http://stacks.math.columbia.edu/

[Ser] Jean-Pierre Serre Local fields, Translated from the French by Marvin Jay Greenberg. Graduate Texts in Mathematics, 67. Springer-Verlag, New York-Berlin, 1979. viii+241 pp.

Statistics and Mathematics Unit, Indian Statistical Institute,, Bangalore, India560059

E-mail address: manish@isibang.ac.in 